\title{
On operator growth and emergent Poincaré symmetries
}

\author{
Javier M. Magán ${ }^{a}$ and Joan Simón ${ }^{b}$ \\ ${ }^{a}$ Instituto Balseiro, Centro Atómico Bariloche, \\ 8400-S.C. de Bariloche, Río Negro, Argentina \\ ${ }^{b}$ School of Mathematics and Maxwell Institute for Mathematical Sciences, \\ University of Edinburgh, \\ Edinburgh EH9 3FD, U.K. \\ E-mail: javier.magan@cab.cnea.gov.ar, j.simon@ed.ac.uk
}

\begin{abstract}
We consider operator growth for generic large-N gauge theories at finite temperature. Our analysis is performed in terms of Fourier modes, which do not mix with other operators as time evolves, and whose correlation functions are determined by their two-point functions alone, at leading order in the large- $\mathrm{N}$ limit. The algebra of these modes allows for a simple analysis of the operators with whom the initial operator mixes over time, and guarantees the existence of boundary CFT operators closing the bulk Poincaré algebra, describing the experience of infalling observers. We discuss several existing approaches to operator growth, such as number operators, proper energies, the many-body recursion method, quantum circuit complexity, and comment on its relation to classical chaos in black hole dynamics. The analysis evades the bulk vs boundary dichotomy and shows that all such approaches are the same at both sides of the holographic duality, a statement that simply rests on the equality between operator evolution itself. In the way, we show all these approaches have a natural formulation in terms of the Gelfand-Naimark-Segal (GNS) construction, which maps operator evolution to a more conventional quantum state evolution, and provides an extension of the notion of operator growth to QFT.
\end{abstract}

Keywords: 1/N Expansion, AdS-CFT Correspondence, Black Holes

ARXIV EPRINT: 2002.03865 


\section{Contents}

1 Introduction 1

2 Operator evolution in large- $\mathrm{N}$ theories 3

3 Emergent Poincaré algebra in holographic theories $\quad 7$

3.1 From Rindler to Poincaré 7

$\begin{array}{lll}3.2 & \text { From boundary CFT to bulk Poincaré algebra } & 10\end{array}$

4 Growth measures $\quad 12$

4.1 Operator growth as state mixing in the GNS construction 13

4.2 Size, number operators and energies in large-N and holographic theories $\quad 16$

$\begin{array}{ll}4.3 \text { The recursion method at large-N } & 18\end{array}$

$\begin{array}{ll}\text { 4.4 Chaos and quantum complexity } & 23\end{array}$

5 Discussion $\quad 26$

A The Gelfand-Naimark-Segal (GNS) construction 28

A.1 GNS of the thermal state 29

A.2 Inner products in the space of operators 31

B Solving the recurrence relation $\quad 33$

\section{Introduction}

In the Schrödinger picture of quantum mechanics, unitary evolution mixes the initial state $|\psi\rangle$ with other quantum states as time evolves

$$
|\psi(t)\rangle=e^{-i H t}|\psi\rangle=\sum_{n=0}^{\infty} \frac{(-i H t)^{n}}{n !}|\psi\rangle \equiv \sum_{n=0}^{\infty} \frac{(-i t)^{n}}{n !}\left|\psi_{n}\right\rangle .
$$

Hence, solving for the time evolution amounts to understanding the states $\left|\psi_{n}\right\rangle \equiv H^{n}|\psi\rangle$, an understanding which is definitely challenging for chaotic Hamiltonians. Similarly, in the Heisenberg picture, unitary evolution mixes the initial operator $\mathcal{O}$ with other operators according to

$$
\mathcal{O}(t)=e^{i H t} \mathcal{O} e^{-i H t}=\sum_{n=0}^{\infty} \frac{(i t)^{n}}{n !}[H, \cdots,[H, \mathcal{O}] \cdots] \equiv \sum_{n=0}^{\infty} \frac{(i t)^{n}}{n !} \mathcal{O}_{n} .
$$

In this case, it is the understanding of the operators $\mathcal{O}_{n} \equiv[H, \cdots,[H, \mathcal{O}] \cdots]$ that allows to solve for the time evolution, and ultimately determines any notion of operator growth one might potentially define. 
The structure of the Heisenberg's time evolution in chaotic systems has attracted some recent interest for several reasons. First, due to its expected connection to quantum chaos. It was found in $[1,2]$, for the case of SYK [3-5], that certain notion of operator size, to be reviewed in the main text, is related to out-of-time-ordered correlation (OTOC) functions [6-8]. Second, because of the relation between operator growth, quantum complexity and the emergence of near horizon symmetries [9-12]. Finally, due to the broader connection between complexity and operator growth, as discussed from different perspectives in $[10,13-15]$, such as using Nielsen's geometric approach to quantum circuit complexity $[16,17]$ or the recursion method in many-body physics [18]. ${ }^{1}$

The main goal of this work is twofold. First, and most importantly, to discuss the structure of the operators $\mathcal{O}_{n}$ in large- $\mathrm{N}$ theories, broadly understood as those theories where large- $\mathrm{N}$ factorization holds [23]. These include large-N holographic theories [24-28]. Second, to revisit some of the existent approaches to operator growth, apply them to large$\mathrm{N}$ theories in the light of our previous analysis, and compare them with quantum circuit complexity and quantum chaos. Within the context of AdS/CFT [29-31], the present approach, based on the analysis of the operators $\mathcal{O}_{n}$, makes manifest that any notion of operator growth is the same at both sides of the duality, given the equivalence of Hilbert spaces, operator algebras, and Heisenberg time evolutions.

Albeit the first objective might seem a hopeless task, given the inherent complexity of a chaotic Hamiltonian, we will show how large- $\mathrm{N}$ factorization and generic finite temperature properties in relativistic QFTs completely determine the action of the operators $\mathcal{O}_{n}$ in most of the relevant states of the theory, at least if the Eigenstate Thermalization Hypothesis (ETH) [32] holds. Since the action of these operators will be shown to be controlled by the 2 -pt function, we conclude that any notion of operator growth is determined by the latter alone, at leading order in the large- $\mathrm{N}$ limit.

As a byproduct of this discussion, we use the operators $\mathcal{O}_{n}$ in holographic theories to construct boundary CFT operators closing the bulk Poincaré algebra. Our construction is analogous to how the Poincaré algebra in free QFT is generated from the algebra of operators in the two Rindler wedges. It also uses the notion of mirror operators introduced in the context of holographic bulk reconstruction [25-27]. This emergent Poincaré algebra controls aspects of bulk infalling physics.

As for the second objective, we first note that, while quantum systems may have different notions of operator size depending on their nature and dynamics, all of them can be formulated as expectation values of simple operators within the Gelfand-Naimark-Segal (GNS) construction. The latter associates a Hilbert space to an algebra of operators, and maps the Heisenberg evolution of operators to the Schrödinger evolution of states in the GNS Hilbert space. This observation allows to extend notions of operator growth to QFT, and large- $\mathrm{N}$ theories in particular.

In this vein, before discussing natural notions of operator growth in QFT, we reformulate different existent notions of operator size in spin systems in terms of the GNS

\footnotetext{
${ }^{1}$ See [19-21] for further work on operator growth in the context of SYK and [22] for a more generic discussion in holographic theories.
} 
Hilbert space. All these notions are given by expectation values of simple operators, such as the number operator. Given this observation, we consider energy and number operators as natural size operators in large- $\mathrm{N}$ holographic theories. In particular, we stress the exponential growth of the proper energy operator [10]. This choice reproduces the upper bound in the Lyapunov exponent in holographic theories and it is intrinsically linked to our discussion of boundary CFT operators closing the bulk Poincaré algebra. Such emergent Poincaré algebra thus reproduces known important physics associated to infalling observers in black holes.

Another existent formalism to study time evolution, mainly in many-body physics, is the so called recursion method [18]. We apply this method to large- $\mathrm{N}$ theories, and find a closed solution for the basis of orthonormalized operators that solves the $1 \mathrm{~d}$ diffusion equation governing the time evolution of operators in this approach. This is accomplished by a change of basis between the operators typically used in the recursion method (the Lanczos basis), and the Fourier mode operators that naturally arise in large- $\mathrm{N}$ theories, which set the ground of our approach.

Finally, we end with a discussion relating Nielsen's geometric approach to quantum circuit complexity, to operator growth and chaos. These are typically viewed and presented as independent concepts, but we observe that when one compares the complexity of formation between a pair of time evolved target states differing by an initial small perturbation, the quantum circuit complexity equals the cost to generate the time evolution of the operator generating the perturbation. Hence, given our previous observations, we can conclude the variation in the quantum circuit complexity in this situation and operator growth must be functionally dependent. On the other hand, the connection between circuit complexity and classical chaos was described in [15], borrowing ideas from [10]. Combining such recent results with our approach to operator evolution in large- $\mathrm{N}$ theories allows for a simple undertanding of the classical chaotic features in black holes found in [33-35].

This work is organized as follows. In section 2, we discuss the operators $\mathcal{O}_{n}$ in large- $\mathrm{N}$ theories. In section 3, we first review the construction of the Poincaré algebra using the algebra of operators in the Rindler wedges in section 3.1. We then apply an analogous construction to holographic large- $\mathrm{N}$ theories in section 3.2. In section 4.1, we reformulate different notions of operator size in the literature using the GNS construction reviewed in appendix A. We discuss natural notions of operator growth and the many-body recursion method in large- $\mathrm{N}$ theories in sections 4.2 and 4.3, respectively. We close with a discussion connecting quantum circuit complexity and operator growth in section 4.4. A summary of our results and the logic purposed in this work are given in section 5 .

\section{Operator evolution in large- $\mathrm{N}$ theories}

The goal of this section is to evaluate the series of nested commutators

$$
\mathcal{O}_{n} \equiv[H, \cdots,[H, \mathcal{O}] \cdots]
$$

controlling the Heisenberg time evolution (1.2) in generic large- $\mathrm{N}$ gauge theories for a subset of initial operators $\mathcal{O}$ for whom large- $\mathrm{N}$ factorization of correlation functions holds [23]. 
Consider a local gauge-invariant scalar operator ${ }^{2} \mathcal{O}(t, \vec{x})$ in a gauge theory defined on $\mathbb{R}^{1, d-1}$. Its Fourier decomposition

$$
\mathcal{O}(t, \vec{x})=\int_{\omega>0} \frac{d \omega d^{d-1} \vec{k}}{(2 \pi)^{d}}\left(\mathcal{O}_{\omega, \vec{k}} e^{-i \omega t+i \vec{k} \vec{x}}+\mathcal{O}_{\omega, \vec{k}}^{\dagger} e^{i \omega t-i \vec{k} \vec{x}}\right)
$$

defines the non-local Fourier mode operators $\mathcal{O}_{\omega, \vec{k}}$ as

$$
\mathcal{O}_{\omega, \vec{k}}=\int d t d^{d-1} \vec{x} \mathcal{O}(t, \vec{x}) e^{i \omega t-i \vec{k} \vec{x}}
$$

with a similar expression for $\mathcal{O}_{\omega, \vec{k}}^{\dagger}$.

Even though the energy $\omega$ and momentum $\vec{k}$ labels are not related to each other by means of a dispersion relation, as it occurs for free quantum fields due to the classical equation of motion, the time evolution of $\mathcal{O}(t, \vec{x})$ remains trivial, as in the latter case. Indeed, the nested commutators (2.1) equal

$$
\mathcal{O}_{n}(t)=i^{-n} \frac{d^{n}}{d t^{n}} \mathcal{O}(t)=\int_{\omega>0} \frac{d \omega d^{d-1} \vec{k}}{(2 \pi)^{d}}\left((-\omega)^{n} \mathcal{O}_{\omega, \vec{k}} e^{-i \omega t+i \vec{k} \vec{x}}+\omega^{n} \mathcal{O}_{\omega, \vec{k}}^{\dagger} e^{i \omega t-i \vec{k} \vec{x}}\right)
$$

In particular, each mode satisfies

$$
\left[H, \mathcal{O}_{\omega, \vec{k}}\right]=-\omega \mathcal{O}_{\omega, \vec{k}} \quad \Longrightarrow \quad \mathcal{O}_{\omega, \vec{k}}(t)=e^{-i \omega t} \mathcal{O}_{\omega, \vec{k}}
$$

and similarly for $\mathcal{O}_{\omega \vec{k}}^{\dagger}$. Hence, these Fourier mode operators do not mix with other operators as time evolves. For this reason, they are especially suited to study operator growth.

The Fourier decomposition (2.2) trades the problem of understanding operator growth, characterized by the operators $\mathcal{O}_{n}$ in (2.4), for the one of understanding the Fourier modes $\mathcal{O}_{\omega, \vec{k}}$ and $\mathcal{O}_{\omega \vec{k}}^{\dagger}$. In general, this is a hard dynamical problem. However, we argue below that these operators are well understood in large- $\mathrm{N}$ gauge theories at finite temperature, where factorization of higher point functions holds.

Indeed, large-N factorization allows to compute higher point correlation functions in terms of the thermal 2-pt functions

$$
\begin{aligned}
& Z_{\beta}^{-1} \operatorname{Tr}\left(e^{-\beta H} \mathcal{O}_{\omega \vec{k}} \mathcal{O}_{\omega^{\prime} \vec{k}^{\prime}}\right)=Z_{\beta}^{-1} \operatorname{Tr}\left(e^{-\beta H} \mathcal{O}_{\omega \vec{k}}^{\dagger} \mathcal{O}_{\omega^{\prime} \vec{k}^{\prime}}^{\dagger}\right)=0 \\
& Z_{\beta}^{-1} \operatorname{Tr}\left(e^{-\beta H} \mathcal{O}_{\omega \vec{k}} \mathcal{O}_{\omega^{\prime} \vec{k}^{\prime}}^{\dagger}\right)=G_{\beta}(\omega, \vec{k}) \delta\left(\omega-\omega^{\prime}\right) \delta^{d-1}\left(\vec{k}-\vec{k}^{\prime}\right) \\
& Z_{\beta}^{-1} \operatorname{Tr}\left(e^{-\beta H} \mathcal{O}_{\omega \vec{k}}^{\dagger} \mathcal{O}_{\omega^{\prime} \vec{k}^{\prime}}\right)=G_{\beta}(-\omega,-\vec{k}) \delta\left(\omega-\omega^{\prime}\right) \delta^{d-1}\left(\vec{k}-\vec{k}^{\prime}\right)
\end{aligned}
$$

where $G_{\beta}(\omega, \vec{k})$ is the Fourier transform of the 2-pt function

$$
\begin{aligned}
G_{\beta}(\omega, \vec{k}) & \equiv \int d t d^{d-1} \vec{x} G_{\beta}(t, \vec{x}) e^{i \omega t-i \vec{k} \vec{x}} \\
& \equiv Z_{\beta}^{-1} \int d t d^{d-1} \vec{x} \operatorname{Tr}\left(e^{-\beta H} \mathcal{O}(t, \vec{x}) \mathcal{O}(0, \overrightarrow{0})\right) e^{i \omega t-i \vec{k} \vec{x}}
\end{aligned}
$$

\footnotetext{
${ }^{2}$ The analysis of more generic smeared operators just follow from linearity as we comment further below.
} 
It follows from (2.6) that the commutators of the Fourier mode operators satisfy

$$
\begin{aligned}
& Z_{\beta}^{-1} \operatorname{Tr}\left(e^{-\beta H}\left[\mathcal{O}_{\omega, \vec{k}}^{\dagger}, \mathcal{O}_{\omega^{\prime}, \vec{k}^{\prime}}^{\dagger}\right]\right)=Z_{\beta}^{-1} \operatorname{Tr}\left(e^{-\beta H}\left[\mathcal{O}_{\omega, \vec{k}}, \mathcal{O}_{\omega^{\prime}, \vec{k}^{\prime}}\right]\right)=0, \\
& Z_{\beta}^{-1} \operatorname{Tr}\left(e^{-\beta H}\left[\mathcal{O}_{\omega, \vec{k}}, \mathcal{O}_{\omega^{\prime}, \vec{k}^{\prime}}^{\dagger}\right]\right)=\left(G_{\beta}(\omega, \vec{k})-G_{\beta}(-\omega,-\vec{k})\right) \delta\left(\omega-\omega^{\prime}\right) \delta^{(d-1)}\left(\vec{k}-\vec{k}^{\prime}\right),
\end{aligned}
$$

up to $1 / N$ corrections. In fact, as stressed in [24] and further developed in [25], due to large- $\mathrm{N}$ factorization, the same statements hold when inserting operators $\mathcal{P}_{1}$ and $\mathcal{P}_{2}$

$$
\begin{aligned}
Z_{\beta}^{-1} \operatorname{Tr}\left(e^{-\beta H} \mathcal{P}_{1}\left[\mathcal{O}_{\omega, \vec{k}}^{\dagger}, \mathcal{O}_{\omega^{\prime}, \vec{k}^{\prime}}^{\dagger}\right] \mathcal{P}_{2}\right)= & Z_{\beta}^{-1} \operatorname{Tr}\left(e^{-\beta H} \mathcal{P}_{1}\left[\mathcal{O}_{\omega, \vec{k}}, \mathcal{O}_{\omega^{\prime}, \vec{k}^{\prime}}\right] \mathcal{P}_{2}\right)=0, \\
Z_{\beta}^{-1} \operatorname{Tr}\left(e^{-\beta H} \mathcal{P}_{1}\left[\mathcal{O}_{\omega, \vec{k}}, \mathcal{O}_{\omega^{\prime}, \vec{k}^{\prime}}^{\dagger}\right] \mathcal{P}_{2}\right)= & \left(G_{\beta}(\omega, \vec{k})-G_{\beta}(-\omega,-\vec{k})\right) \delta\left(\omega-\omega^{\prime}\right) \delta^{(d-1)}\left(\vec{k}-\vec{k}^{\prime}\right) . \\
& \cdot \operatorname{Tr}\left(e^{-\beta H} \mathcal{P}_{1} \mathcal{P}_{2}\right)
\end{aligned}
$$

involving a number of legs not scaling with $N$. Therefore, these commutators behave as cnumbers when inserted in correlation functions within this regime, a characteristic feature of free fields.

Correlators (2.6) and (2.9), together with linearity, allow us to evaluate the expectation values of the nested commutators (2.4) in the thermal ensemble at any temperature. However, for the subset of large-N gauge theories satisfying the Eigenstate Thermalization Hypothesis (ETH) [32], the set of states in the Hilbert space where the previous correlation functions hold is much larger. This is because ETH ensures the same expectation values apply to most energy eigenstates compatible with the physical temperature. Therefore, in the large- $\mathrm{N}$ limit, correlators (2.6) and (2.9) define the action of the operators $\mathcal{O}_{\omega, \vec{k}}, \mathcal{O}_{\omega, \vec{k}}^{\dagger}$, and consequently the action of the $\mathcal{O}_{n}$ operators, in the basis of eigenstates of the theory, up to a set of atypical energy eigenstates. ${ }^{3}$ It is the knowledge of this action, and the associated expectation values, that actually defines these operators in this limit. Following the logic in this work, this information determines the time evolution of the initial operator $\mathcal{O}(t, \vec{x})$ and any notion of operator growth associated with it. ${ }^{4}$

Let us stress that, mathematically, the correlators (2.6), (2.8) and (2.8) assume the energy-momentum labels do not scale with $N$. However, physically, these high energy modes are not necessary. Since local operators (2.2) have an infinite amount of energy, the actual physical operators are smeared versions of these

$$
\mathcal{O}=\int d t d^{d-1} \vec{x} f(x, t) \mathcal{O}(t, \vec{x})=\int_{\omega>0} \frac{d \omega d^{d-1} \vec{k}}{(2 \pi)^{d}}\left(\tilde{f}(\omega, \vec{k}) \mathcal{O}_{\omega, \vec{k}}+\tilde{f}^{*}(\omega, \vec{k}) \mathcal{O}_{\omega, \vec{k}}^{\dagger}\right)
$$

where

$$
\tilde{f}(\omega, \vec{k}) \equiv \int d t d^{d-1} \vec{x} f(t, \vec{x}) e^{i \omega t-i \vec{k} \vec{x}} .
$$

To have a well defined (finite energy) operator $\mathcal{O}$, the smoothness of the smearing function $f(t, \vec{x})$ must not blow up in the large-N limit. The Fourier transform of such function will

\footnotetext{
${ }^{3}$ For a discussion on large- $\mathrm{N}$ factorization in chaotic theories and its relevance to ETH, see [25, 28, 36].

${ }^{4}$ This is typical in probability theory. We can define a random variable by its associated probability distribution, or equivalently by giving all its moments. From a physical perspective, the second option is better since the moments are the ones being measured.
} 
exponentially suppress the modes $\mathcal{O}_{\omega, \vec{k}}$ with frequencies and wavelengths scaling with $N$. This smearing condition, together with large- $\mathrm{N}$ factorization, provides a good and precise definition of a "simple" operator in large-N QFT's. ${ }^{5}$

Before ending this section, it is worth making a couple of closing remarks. First, the "growth" of the operator (2.2) in space, as defined by the nested commutators

$$
\begin{aligned}
{\left[P^{j}, \cdots,\left[P^{j}, \mathcal{O}(t, \vec{x})\right] \cdots\right] } & =(-i)^{n} \frac{d^{n}}{d x_{j}^{n}} \mathcal{O}(t, \vec{x}) \\
& =\int_{\omega>0} \frac{d \omega d^{d-1} \vec{k}}{(2 \pi)^{d}}\left[k_{j}^{n} \mathcal{O}_{\omega, \vec{k}} e^{-i \omega t+i \vec{k} \vec{x}}+\left(-k_{j}\right)^{n} \mathcal{O}_{\omega, \vec{k}}^{\dagger} e^{i \omega t-i \vec{k} \vec{x}}\right] .
\end{aligned}
$$

is also determined by the same 2-pt functions above. Second, the generators of time $(H)$ and space $(\vec{P})$ translations reduce to

$$
H=\int_{\omega>0} \frac{d \omega d^{d-1} \vec{k}}{(2 \pi)^{d}} \omega \mathcal{O}_{\omega, \vec{k}}^{\dagger} \mathcal{O}_{\omega, \vec{k}} \quad \text { and } \quad \vec{P}=\int_{\omega>0} \frac{d \omega d^{d-1} \vec{k}}{(2 \pi)^{d}} \vec{k} \mathcal{O}_{\omega, \vec{k}}^{\dagger} \mathcal{O}_{\omega, \vec{k}}
$$

in the large- $N$ limit we are considering.

Modular time evolution. Besides unitary time evolution, there is a second natural notion of evolution, modular time evolution, when restricting physics to subregions of spacetime (see [37] for a review). Modular time evolution is defined as the unitary evolution generated by the modular hamiltonian $H_{\text {mod }}$ in the region of interest

$$
\mathcal{O}(s) \equiv e^{i s H_{\bmod }} \mathcal{O} e^{-i s H_{\bmod }}=\rho^{-i s} \mathcal{O} \rho^{i s},
$$

where $H_{\text {mod }}$ is related to the reduced density matrix ${ }^{6}$ in this region $\rho$, as $\rho=e^{-H_{\text {mod }}}$.

It is interesting to ask for the structure of operator evolution in this context. ${ }^{7}$ Since

$$
\mathcal{O}(s)=e^{i s H_{\text {mod }}} \mathcal{O} e^{-i s H_{\text {mod }}}=\sum_{n=0}^{\infty} \frac{(i s)^{n}}{n !}\left[H_{\text {mod }}, \cdots,\left[H_{\text {mod }}, \mathcal{O}\right] \cdots\right] \equiv \sum_{n=0}^{\infty} \frac{(i s)^{n}}{n !} \mathcal{O}_{n}^{\text {mod }},
$$

this structure is determined by the operators

$$
\mathcal{O}_{n}^{\bmod } \equiv\left[H_{\text {mod }}, \cdots,\left[H_{\text {mod }}, \mathcal{O}\right] \cdots\right],
$$

which are the only ones with whom the initial operator mixes through modular time evolution. Proceeding as before, we can Fourier transform the fields, but against modular time evolution

$$
\mathcal{O}_{\omega}=\int d s \mathcal{O}(s) e^{i \omega s}
$$

This allows for a simple computation of the action of $\mathcal{O}_{n}^{\text {mod }}$ in all eigenstates of the theory, in the vein of (2.4), if the correlators of the modular field modes are gaussian, as expected for holographic theories with free bulk duals.

\footnotetext{
${ }^{5}$ In the context of spin systems, a "simple" operator is defined as one involving the product of an $\mathcal{O}(1)$ number of spins.

${ }^{6}$ In QFT care has to be taken when defining these objects, but modular time evolution is well and unambiguously defined, see [37] and [38] for example.

${ }^{7}$ Operator growth in the context of modular time evolution has also been considered recently in [39].
} 


\section{$3 \quad$ Emergent Poincaré algebra in holographic theories}

The arguments of the previous section apply, as a particular case, to large-N holographic theories. For such theories, the dual bulk description to the CFT at finite temperature is a black hole [40]. The spectrum of low energy bulk excitations is captured by a small (not scaling with $N$ ) number of boundary generalized free fields, whose Fourier modes satisfy similar commutation relations to the ones in (2.9) (see below for a more precise account). It follows from section 2 that the time evolution of these boundary operators is controlled by thermal 2-pt functions, up to $1 / N$ corrections.

Consider now low energy bulk excitations near the black hole horizon. The geometry they probe is locally equivalent to a Rindler horizon. We can ask whether the exact relations satisfied by 2-pt functions in a fixed Rindler spacetime, characterizing the differences between infalling and uniformly accelerated observers in free QFT, will continue to hold at lowest order in a $1 / N$ expansion in the holographic set-up. Similarly, we can ask whether we can see the relations between infalling and acelerated observers directly in the CFT description. The objective of this section is to provide a constructive answer to these questions, just based on the observations made in the previous section.

More concretely, our logic will proceed as follows. Free QFT in Rindler spacetime is Poincaré invariant. Motivated by the black hole scenario and the questions above, we can ask how to build operators closing an exact Poincaré algebra out of the algebra of operators existing in the right Rindler wedge alone. We will review such construction in section 3.1, stressing it simply relies on the algebra satisfied by the Rindler creation/annihilation operators. But this is the same algebra satisfied by the Fourier modes of the generalized free fields, dual to the bulk excitations in the boundary CFT. Therefore, we will conclude that applying the same Rindler construction to large- $\mathrm{N}$ holographic theories gives rise to boundary CFT operators closing the bulk Poincaré algebra at lowest order in a $1 / N$ expansion. The implications of this construction for operator growth will be discussed in the next section.

\subsection{From Rindler to Poincaré}

Consider a free quantum scalar field of mass $m$ in $\mathbb{R}^{1, d}$. We want to review how to construct the relevant generators of the Poincaré algebra starting from the operator algebra in the right Rindler wedge plus the associated thermal state on it. To set some notation, let us decompose the space directions into $z$ and $\vec{x}$, with conjugate momentum $k_{z}$ and $\vec{k}$, respectively, so that $z$ corresponds to the direction along which the Rindler observer is uniformly accelerated. Local fields in the right Rindler patch can be expanded in terms of creation and annihilation operators

$$
\hat{a}_{\omega \vec{k}}^{\mathrm{R}}, \quad \hat{a}_{\omega \vec{k}}^{\mathrm{R} \dagger}
$$

satisfying the standard commutation relations

$$
\left[\hat{a}_{\omega \vec{k}}^{\mathrm{R}}, \hat{a}_{\omega^{\prime} \vec{k}^{\prime}}^{R \dagger}\right]=\delta\left(\omega-\omega^{\prime}\right) \delta^{(d-1)}\left(\vec{k}-\vec{k}^{\prime}\right)
$$


Since $\omega$ stands for the Rindler energy, time evolution in this Rindler wedge is generated by the operator

$$
\hat{H}^{\mathrm{R}}=\int d \omega d \vec{k} \omega \hat{a}_{\omega \vec{k}}^{\mathrm{R} \dagger} \hat{a}_{\omega \vec{k}}^{\mathrm{R}} .
$$

It follows that each operator $\hat{a}_{\omega \vec{k}}^{\mathrm{R}}$ evolves as

$$
\left[\hat{H}^{\mathrm{R}}, \hat{a}_{\omega \vec{k}}^{\mathrm{R}}\right]=-\omega \hat{a}_{\omega \vec{k}}^{\mathrm{R}} \Rightarrow \hat{a}_{\omega \vec{k}}^{\mathrm{R}}(t)=e^{-i \omega t} \hat{a}_{\omega \vec{k}}^{\mathrm{R}}
$$

as usual in free quantum field theory, with a similar expression for $\hat{a}_{\omega \vec{k}}^{\mathrm{R} \dagger}(t)$. Linearity extends these claims to local quantum fields. Notice Rindler time is labelled as $t$. Minkowski operators and coordinates will carry an $M$ superscript.

Consider a thermal state $\rho_{\beta}$ in the right Rindler wedge quantum field theory (QFT) with $\beta=\frac{2 \pi}{a}$, where $a$ stands for the proper acceleration defining the Rindler frame. The state $\rho_{\beta}$ is well known to be the reduced density matrix of the pure state $\left|0_{\mathrm{M}}\right\rangle$, i.e. the vacuum of the QFT in Minkowski (see [41] for a review and more details about QFT in Rindler space). This purification involves a duplication of the operator algebra giving rise to a new set of operators

$$
\hat{a}_{\omega \vec{k}}^{\mathrm{L}}, \hat{a}_{\omega \vec{k}}^{\mathrm{L \dagger}}
$$

corresponding to the creation and annihilation operators in the left Rindler wedge from the perspective of the full Minkowski spacetime. These operators commute with the original ones (3.2) and altogether form a complete basis of operators in the Minkowski Hilbert space. This matches the general Gelfand-Naimark-Segal (GNS) representation of thermal states reviewed in appendix A, for later convenience. In this appendix, the origin of the duplication of the algebra responsible for the canonical purification of the thermal Rindler density matrix $\rho_{\beta}$ in the current discussion is explained in detail.

The Minkowski vacuum $\left|0_{\mathrm{M}}\right\rangle$ is determined by the relations

$$
\left(\hat{a}_{\omega \vec{k}}^{\mathrm{L}}-e^{-\pi \frac{\omega}{a}} \hat{a}_{\omega(-\vec{k})}^{\mathrm{R} \dagger}\right)\left|0_{\mathrm{M}}\right\rangle=\left(\hat{a}_{\omega \vec{k}}^{\mathrm{R}}-e^{-\pi \frac{\omega}{a}} \hat{a}_{\omega(-\vec{k})}^{\mathrm{L} \dagger}\right)\left|0_{\mathrm{M}}\right\rangle=0 .
$$

These allow to write the action of both $\hat{a}_{\omega \vec{k}}^{\mathrm{L}}$ and $\hat{a}_{\omega \vec{k}}^{\mathrm{L} \dagger}$ on $\left|0_{\mathrm{M}}\right\rangle$ in terms of operators acting on the right wedge. Notice that in our conventions, those of ref. [41], Rindler time in the left wedge also runs in the same direction as in the right Rindler wedge. To get the opposite conventions typically used in black hole physics and holography [42], one needs to perform the replacement $\hat{a}_{\omega(-\vec{k})}^{\mathrm{L} \dagger} \rightarrow \hat{a}_{\omega \vec{k}}^{\mathrm{L} \dagger}$.

Given this complete basis of operators, there are two additional bases one can introduce which will be relevant in what follows. The first is the set of creation and annihilation operators associated with Minkowski time evolution

$$
\begin{aligned}
\hat{a}_{k_{z} \vec{k}}^{\mathrm{M}}= & \int_{0}^{\infty} \frac{d \omega}{\sqrt{2 \pi a \omega_{\vec{k}}}} \frac{1}{\sqrt{1-e^{-2 \pi \omega / a}}} \\
& \times\left[e^{i \vartheta\left(k_{z}\right) \frac{\omega}{a}}\left(\hat{a}_{\omega \vec{k}}^{\mathrm{L}}-e^{-\pi \frac{\omega}{a}} \hat{a}_{\omega(-\vec{k})}^{\mathrm{R} \dagger}\right)+e^{-i \vartheta\left(k_{z}\right) \frac{\omega}{a}}\left(\hat{a}_{\omega \vec{k}}^{\mathrm{R}}-e^{-\pi \frac{\omega}{a}} \hat{a}_{\omega(-\vec{k})}^{\mathrm{L} \dagger}\right)\right],
\end{aligned}
$$

where

$$
\vartheta\left(k_{z}\right)=\frac{1}{2} \log \left(\frac{\omega_{\vec{k}}+k_{z}}{\omega_{\vec{k}}-k_{z}}\right)
$$


is the standard rapidity in relativistic physics and $\omega_{\vec{k}}^{2}=m^{2}+k_{z}^{2}+|\vec{k}|^{2}$ is the on-shell Minkowski frequency carried by each mode. In this basis, the generators of Minkoswki time and spatial $z$ translations are the standard expressions

$$
\begin{aligned}
\hat{H}^{\mathrm{M}} & =\int d k_{z} d^{d-1} \vec{k} \omega_{\vec{k}} \hat{a}_{k_{z} \vec{k}}^{\mathrm{M} \dagger} \hat{a}_{k_{z} \vec{k}}^{\mathrm{M}}, \\
\hat{P}_{z}^{\mathrm{M}} & =\int d k_{z} d^{d-1} \vec{k} k_{z} \hat{a}_{k_{z} \vec{k}}^{\mathrm{M} \dagger} \hat{a}_{k_{z} \vec{k}}^{\mathrm{M}},
\end{aligned}
$$

whereas the number operator equals

$$
\hat{N}^{\mathrm{M}}=\int d k_{z} d^{d-1} \vec{k} \hat{a}_{k_{z} \vec{k}}^{\mathrm{M} \dagger} \hat{a}_{k_{z} \vec{k}}^{\mathrm{M}} .
$$

Notice that using (3.6), any Minkowski mode can be easily generated by acting with operators in the right wedge

$$
\hat{a}_{k_{z} \vec{k}}^{\mathrm{M} \dagger}\left|0_{M}\right\rangle=\int_{0}^{\infty} \frac{d \omega}{\sqrt{2 \pi a \omega_{\vec{k}}}} \sqrt{2 \sinh \frac{\pi \omega}{a}}\left[e^{-i \frac{\omega}{a}\left(\vartheta\left(k_{z}\right)+i \frac{\pi}{2}\right)} \hat{a}_{\omega(-\vec{k})}^{R}+e^{i \frac{\omega}{a}\left(\vartheta\left(k_{z}\right)+i \frac{\pi}{2}\right)} \hat{a}_{\omega \vec{k}}^{R \dagger}\right]\left|0_{\mathrm{M}}\right\rangle .
$$

The second additonal basis is the Unruh basis, defined by an appropriate normalisation of the operators annihilating $\left|0_{\mathrm{M}}\right\rangle$ in (3.6)

$$
\begin{aligned}
& b_{+\omega, \vec{k}}=\frac{1}{\sqrt{1-e^{-2 \pi \frac{\omega}{a}}}}\left(\hat{a}_{\omega \vec{k}}^{\mathrm{L}}-e^{-\pi \frac{\omega}{a}} \hat{a}_{\omega(-\vec{k})}^{\mathrm{R} \dagger}\right), \\
& b_{-\omega, \vec{k}}=\frac{1}{\sqrt{1-e^{-2 \pi \frac{\omega}{a}}}}\left(\hat{a}_{\omega \vec{k}}^{\mathrm{R}}-e^{-\pi \frac{\omega}{a}} \hat{a}_{\omega(-\vec{k})}^{\mathrm{L \dagger}}\right),
\end{aligned}
$$

together with $b_{ \pm \omega, \vec{k}}^{\dagger}$. These operators satisfy standard commutation relations

$$
\left[\hat{b}_{ \pm \omega \vec{k}}, \hat{b}_{ \pm \omega \vec{k}}^{\dagger}\right]=\delta\left(\omega-\omega^{\prime}\right) \delta\left(\vec{k}-\overrightarrow{k^{\prime}}\right) .
$$

They are convenient to determine the thermal nature of the Rindler modes in $\left|0_{\mathrm{M}}\right\rangle$ [43]. Indeed, using

$$
\hat{a}_{\omega \vec{k}}^{\mathrm{R}}=\frac{\hat{b}_{-\omega \vec{k}}+e^{-\pi \frac{\omega}{a}} \hat{b}_{+\omega-\vec{k}}^{\dagger}}{\sqrt{1-e^{-2 \pi \frac{\omega}{a}}}}, \quad \hat{a}_{\omega \vec{k}}^{\mathrm{L}}=\frac{\hat{b}_{+\omega \vec{k}}+e^{-\pi \frac{\omega}{a}} \hat{b}_{-\omega-\vec{k}}^{\dagger}}{\sqrt{1-e^{-2 \pi \frac{\omega}{a}}}},
$$

and the fact that Unruh modes annihilate $\left|0_{\mathrm{M}}\right\rangle$ (eq. (3.6)), one easily finds

$$
\begin{aligned}
& \left\langle 0_{\mathrm{M}}\left|\hat{a}_{\omega \vec{k}}^{\mathrm{R}} \hat{a}_{\omega \vec{k}}^{\mathrm{R}}\right| 0_{\mathrm{M}}\right\rangle=\left\langle 0_{\mathrm{M}}\left|\hat{a}_{\omega \vec{k}}^{\mathrm{R} \dagger} \hat{a}_{\omega \vec{k}}^{\mathrm{R} \dagger}\right| 0_{\mathrm{M}}\right\rangle=0, \\
& \left\langle 0_{\mathrm{M}}\left|\hat{a}_{\omega \vec{k}}^{\mathrm{R}} \hat{a}_{\omega \vec{k}}^{\mathrm{R} \dagger}\right| 0_{\mathrm{M}}\right\rangle=\frac{e^{\beta \omega}}{e^{\beta \omega}-1} \delta\left(\omega-\omega^{\prime}\right) \delta^{(d-1)}\left(\vec{k}-\vec{k}^{\prime}\right), \\
& \left\langle 0_{\mathrm{M}}\left|\hat{a}_{\omega \vec{k}}^{\mathrm{R} \dagger} \hat{a}_{\omega \vec{k}}^{\mathrm{R}}\right| 0_{\mathrm{M}}\right\rangle=\frac{1}{e^{\beta \omega}-1} \delta\left(\omega-\omega^{\prime}\right) \delta^{(d-1)}\left(\vec{k}-\vec{k}^{\prime}\right) .
\end{aligned}
$$

The associated Unruh number and energy operators, labelled with a $U$ superscript, are

$$
\begin{aligned}
& \hat{H}^{\mathrm{U}}=\int_{0}^{\infty} d \omega \int d^{d-1} \vec{k} \omega\left[\hat{b}_{\omega \vec{k}}^{\dagger} \hat{b}_{\omega \vec{k}}+\hat{b}_{-\omega \vec{k}}^{\dagger} \hat{b}_{-\omega \vec{k}}\right], \\
& \hat{N}^{\mathrm{U}}=\int_{0}^{\infty} d \omega \int d^{d-1} \vec{k}\left[\hat{b}_{\omega \vec{k}}^{\dagger} \hat{b}_{\omega \vec{k}}+\hat{b}_{-\omega \vec{k}}^{\dagger} \hat{b}_{-\omega \vec{k}}\right] .
\end{aligned}
$$

It can be verified by direct computation that $\hat{N}^{\mathrm{U}}=\hat{N}^{\mathrm{M}}$. 
We now have all the required ingredients to construct the generators of the Poincaré algebra. Indeed, the operators (3.9) generate time and space translations, whereas the total Rindler Hamiltonian, in appropriate units,

$$
\hat{K}_{\mathrm{z}}^{\mathrm{M}}=\frac{1}{a}\left(\hat{H}_{\mathrm{R}}-\hat{H}_{\mathrm{L}}\right)
$$

gives rise to the boost operator. Let us stress that we view all three operators as implicitly (or indeed explicitly) defined in terms of the right Rindler wedge operators satisfying the commutation relations (3.2), and placed in thermal state $\rho_{\beta}$. Using these and the definition of the Minkowski creation/annihilation operators (3.7), one can verify $\hat{H}^{\mathrm{M}}, \hat{P}_{\mathrm{z}}^{\mathrm{M}}$ and $\hat{K}_{\mathrm{z}}^{\mathrm{M}}$ satisfy the commutation relations characteristic of the Poincaré algebra

$$
\left[\hat{H}^{\mathrm{M}}, \hat{K}_{\mathrm{z}}^{\mathrm{M}}\right]=i \hat{P}_{\mathrm{z}}^{\mathrm{M}}, \quad\left[\hat{P}_{\mathrm{z}}^{\mathrm{M}}, \hat{K}_{\mathrm{z}}^{\mathrm{M}}\right]=-i \hat{H}^{\mathrm{M}} .
$$

To summarize, using the free algebra of creation and annihilation operators in the right Rindler wedge, the canonical duplication of the algebra in the thermal state, and the definition of the Minkowski vacuum (3.6), we constructed a set of operators $\hat{H}^{\mathrm{M}}, \hat{P}_{z}^{\mathrm{M}}$ and $\hat{K}_{\mathrm{z}}^{\mathrm{M}}$, implicitly defined using (3.7) and its hermitian conjugate, closing the exact Poincaré algebra.

\subsection{From boundary CFT to bulk Poincaré algebra}

Let us consider large-N holographic theories. Due to large-N factorization, thermal correlation functions are determined by (2.6) and (2.9). As noticed in [25], a normalised version of the Fourier operators appearing in section 2

$$
\hat{\mathcal{O}}_{\omega, \vec{k}} \equiv \frac{\mathcal{O}_{\omega, \vec{k}}}{\left(G_{\beta}(\omega, \vec{k})-G_{\beta}(-\omega,-\vec{k})\right)^{1 / 2}}=\frac{1}{\sqrt{G_{\beta}(t, \vec{k})}} \frac{\mathcal{O}_{\omega, \vec{k}}}{\sqrt{1-e^{-\beta \omega}}},
$$

have canonical commutations relations and their thermal expectation values satisfy

$$
\begin{aligned}
& Z_{\beta}^{-1} \operatorname{Tr}\left(e^{-\beta H} \hat{\mathcal{O}}_{\omega, \vec{k}}^{\dagger} \hat{\mathcal{O}}_{\omega^{\prime}, \vec{k}^{\prime}}\right)=\frac{1}{e^{\beta \omega}-1} \delta\left(\omega-\omega^{\prime}\right) \delta^{d-1}\left(\vec{k}-\vec{k}^{\prime}\right), \\
& Z_{\beta}^{-1} \operatorname{Tr}\left(e^{-\beta H} \hat{\mathcal{O}}_{\omega, \vec{k}} \hat{\mathcal{O}}_{\omega^{\prime}, \vec{k}^{\prime}}^{\dagger}\right)=\frac{e^{\beta \omega}}{e^{\beta \omega}-1} \delta\left(\omega-\omega^{\prime}\right) \delta^{d-1}\left(\vec{k}-\vec{k}^{\prime}\right) .
\end{aligned}
$$

Hence, these operators display the same algebra and expectation values as the right Rindler wedge creation/annihilation operators in (3.2) and (3.15). We use this observation to explicitly construct boundary CFT operators closing the bulk Poincaré algebra, up to $1 / N$ corrections, for both 2-sided and 1-sided holographic AdS black holes.

2-sided AdS black holes. If the state in the (right) CFT is exactly thermal, we can canonically purify it by the associated thermofield double state

$$
|\mathrm{TFD}\rangle \equiv \frac{1}{\sqrt{Z(\beta)}} \sum_{i} e^{-\frac{\beta E_{i}}{2}}\left|E_{i}\right\rangle_{\mathrm{L}} \otimes\left|E_{i}\right\rangle_{\mathrm{R}}
$$

belonging to the duplicated Hilbert space $\mathcal{H}_{\mathrm{L}} \otimes \mathcal{H}_{\mathrm{R}}$. This pure state matches the GNS construction of the thermal state reviewed in appendix A.1. It is holographically dual to 
the 2-sided eternal AdS black hole [42]. Since the algebra of operators is also duplicated, it gives rise to two sets of commuting modes $\mathcal{O}_{\mathrm{L} \omega, \vec{k}}^{\dagger}$ and $\mathcal{O}_{\mathrm{R} \omega, \vec{k}}^{\dagger}$ satisfying the same algebra and with the same expectation values as in (2.6) and (2.9). They also verify the relation

$$
\left(\hat{\mathcal{O}}_{\mathrm{L} \omega, \vec{k}}-e^{-\frac{\beta \omega}{2}} \hat{\mathcal{O}}_{\mathrm{R} \omega, \vec{k}}^{\dagger}\right)|\mathrm{TFD}\rangle=\left(\hat{\mathcal{O}}_{\mathrm{R} \omega, \vec{k}}-e^{-\frac{\beta \omega}{2}} \hat{\mathcal{O}}_{\mathrm{L} \omega, \vec{k}}^{\dagger}\right)|\mathrm{TFD}\rangle=0 .
$$

The origin of this equation is explained in appendix A.1. It is an special case of eq. (A.18), and it is basically equivalent to (3.6), the equation defining $\left|0_{\mathrm{M}}\right\rangle$ in the Rindler discussion. As stressed there, our conventions involve time running in the same direction in both wedges. Furthermore, the Rindler acceleration $a$ is mapped to the black hole temperature using $\beta=\frac{2 \pi}{a}$.

Given the equivalence between the algebra of left/right creation/annihilation operators in Rindler, and the left $\hat{\mathcal{O}}_{\mathrm{L} \omega, \vec{k}}, \hat{\mathcal{O}}_{\mathrm{L} \omega, \vec{k}}^{\dagger}$ and right $\hat{\mathcal{O}}_{\mathrm{R} \omega, \vec{k}}, \hat{\mathcal{O}}_{\mathrm{R} \omega, \vec{k}}^{\dagger}$ Fourier modes belonging to the two boundary CFT Hilbert spaces, we can now proceed analogously to our discussion of the different bases of operators and Poincaré generators associated to Rindler physics. In particular, the generator of time translations in the right CFT reduces in the large-N limit to

$$
\hat{H}^{\mathrm{R}}=\int d \omega d \vec{k} \omega \hat{\mathcal{O}}_{\mathrm{R} \omega, \vec{k}}^{\dagger} \hat{\mathcal{O}}_{\mathrm{R} \omega, \vec{k}} .
$$

The Unruh creation/annihilation operators can be defined by

$$
\begin{aligned}
& \mathcal{O}_{+\omega, \vec{k}}^{\mathrm{U}}=\frac{1}{\sqrt{1-e^{-\beta \omega}}}\left(\hat{\mathcal{O}}_{\mathrm{L} \omega, \vec{k}}-e^{-\beta \omega / 2} \hat{\mathcal{O}}_{\mathrm{R} \omega, \vec{k}}^{\dagger}\right), \\
& \mathcal{O}_{-\omega, \vec{k}}^{\mathrm{U}}=\frac{1}{\sqrt{1-e^{-\beta \omega}}}\left(\hat{\mathcal{O}}_{\mathrm{R} \omega, \vec{k}}-e^{-\beta \omega / 2} \hat{\mathcal{O}}_{\mathrm{L} \omega, \vec{k}}^{\dagger}\right),
\end{aligned}
$$

while Minkowski annihilation modes can be defined by

$$
\begin{aligned}
\mathcal{O}_{k_{z} \vec{k}}^{\mathrm{M}}= & \int_{0}^{\infty} \frac{d \omega}{\sqrt{2 \pi a k_{0}}} \frac{1}{\sqrt{1-e^{-\beta \omega}}} \\
& \times\left[e^{i 2 \pi \vartheta\left(k_{z}\right) \omega / \beta}\left(\hat{\mathcal{O}}_{\mathrm{L} \omega, \vec{k}}-e^{-\beta \omega / 2} \hat{\mathcal{O}}_{\mathrm{R} \omega, \vec{k}}^{\dagger}\right)+e^{-i 2 \pi \vartheta\left(k_{z}\right) \omega / \beta}\left(\hat{\mathcal{O}}_{\mathrm{R} \omega, \vec{k}}-e^{-\beta \omega / 2} \hat{\mathcal{O}}_{\mathrm{L} \omega, \vec{k}}^{\dagger}\right)\right]
\end{aligned}
$$

These operators allow us to define operators generating Minkowski time and spatial $z$ translations by

$$
\begin{aligned}
\hat{H}^{\mathrm{M}} & =\int d k_{z} d^{d-1} \vec{k} \omega_{\vec{k}} \mathcal{O}_{k_{z} \vec{k}}^{\mathrm{M} \dagger} \mathcal{O}_{k_{z} \vec{k}}^{\mathrm{M}} \\
\hat{P}_{z}^{\mathrm{M}} & =\int d k_{z} d^{d-1} \vec{k} k_{z} \mathcal{O}_{k_{z} \vec{k}}^{\mathrm{M} \dagger} \mathcal{O}_{k_{z} \vec{k}}^{\mathrm{M}},
\end{aligned}
$$

where $\omega_{\vec{k}}^{2}=m^{2}+k_{z}^{2}+|\vec{k}|^{2}$. The associated number operator is just given by

$$
\hat{N}^{\mathrm{M}}=\int d k_{z} d^{d-1} \vec{k} \mathcal{O}_{k_{z} \vec{k}}^{M \dagger} \mathcal{O}_{k_{z} \vec{k}}^{M}
$$

Since the algebra of the modes $\hat{\mathcal{O}}_{\mathrm{R} \omega, \vec{k}}$ and $\hat{\mathcal{O}}_{\mathrm{L} \omega, \vec{k}}$, together with the expectation values in the thermofield double, are equal to the ones in the Rindler discussion, up to $1 / N$ corrections, we conclude these boundary CFT operators close the same bulk Poincaré algebra

$$
\left[\hat{H}^{\mathrm{M}}, \hat{H}^{\mathrm{T}}\right]=i \hat{P}_{z}^{\mathrm{M}}, \quad\left[\hat{P}^{\mathrm{M}}, \hat{H}^{\mathrm{T}}\right]=-i \hat{H}^{\mathrm{M}},
$$


where the boost operator $\hat{H}^{\mathrm{T}}$ is the total boost Hamiltonian of the two decoupled CFTs defined by

$$
\hat{H}^{\mathrm{T}}=\frac{\beta}{2 \pi}\left(\hat{H}^{\mathrm{R}}-\hat{H}^{\mathrm{L}}\right)
$$

This is the same as in the Rindler discussion (3.17), after the replacement $a \rightarrow 2 \pi / \beta$.

1-sided AdS black holes. The proposal on bulk reconstruction of the black hole interior developed in [25-27] extends the previous discussion to single AdS black holes involving a single boundary CFT. Observing that energy eigenstates $\left|E_{i}\right\rangle$ are well approximated by the thermal ensemble, one can work within the code subspace $[44,45]$ and show that $\left|E_{i}\right\rangle$ is a cyclic and a separating vector in the Hilbert space with respect to the code subspace algebra $[28,36]$. The Tomita-Takesaki theorem ${ }^{8}$ guarantees the existence of a non-trivial "mirror" commutant. The mirror operators $\tilde{O}_{\omega, \vec{k}}$ generating this commutant play a similar role to the operators $\mathcal{O}_{\mathrm{L} \omega, \vec{k}}$ in the 2-sided discussion, but they belong to the same boundary CFT dual to the single AdS black hole. Mirror operators are state dependent and defined by the relations

$$
\begin{aligned}
\tilde{\mathcal{O}}_{\omega, \vec{k}}\left|\Psi_{i}\right\rangle & =e^{-\frac{\beta \omega}{2}} \mathcal{O}_{\omega, \vec{k}}^{\dagger}\left|\Psi_{i}\right\rangle \\
\tilde{\mathcal{O}}_{\omega, \vec{k}} \mathcal{O}_{\omega_{1}, \vec{k}_{1}} \ldots \mathcal{O}_{\omega_{n}, \vec{k}_{n}}\left|\Psi_{i}\right\rangle & =\mathcal{O}_{\omega_{1}, \vec{k}_{1}} \ldots \mathcal{O}_{\omega_{n}, \vec{k}_{n}} \tilde{\mathcal{O}}_{\omega, \vec{k}}\left|\Psi_{i}\right\rangle, \\
{\left[H, \tilde{\mathcal{O}}_{\omega, \vec{k}}\right] \mathcal{O}_{\omega_{1}, \vec{k}_{1}} \ldots \mathcal{O}_{\omega_{n}, \vec{k}_{n}}\left|\Psi_{i}\right\rangle } & =\omega \tilde{\mathcal{O}}_{\omega, \vec{k}} \mathcal{O}_{\omega_{1}, \vec{k}_{1}} \ldots \mathcal{O}_{\omega_{n}, \vec{k}_{n}}\left|\Psi_{i}\right\rangle,
\end{aligned}
$$

which only hold within the code subspace. As discussed in [28], there are some ambiguities regarding the $1 / N$ extension of these operators, but for us it will be enough to work within the code subspace. This, together with microstates $\left|E_{i}\right\rangle$ being well approximated by the canonical ensemble, ensures the defining properties (3.30) give rise to an algebra and correlation functions that are equivalent to the algebra of the $\mathcal{O}_{\mathrm{L} \omega, \vec{k}}$ in the 2-sided discussion, and therefore to the algebra of annihilation operators in the left wedge. We can thus proceed anagolously to the 2 -sided discussion. In particular we can define the different basis and generators, including the Poincaré ones, by the very same formulas, just changing the modes $\mathcal{O}_{\mathrm{L} \omega, \vec{k}}$ by the mirror modes $\tilde{O}_{\omega, \vec{k}}$.

Let us summarize the results of this section. Building on the transparent construction in Rindler spacetime, where the Poincaré algebra is seen to directly rest on the algebra of operators in the right wedge (together with an appropriate thermal state), we have established the existence of CFT operators in large-N CFTs closing the bulk Poincaré algebra both in the 2-sided and 1-sided black hole scenarios. This algebra describes the experience of infalling observers in black holes.

\section{Growth measures}

As argued in section 2, time evolution of simple perturbations in large- $\mathrm{N}$ theories at any finite temperature is captured by the Fourier modes $\mathcal{O}_{\omega, \vec{k}}$ and $\mathcal{O}_{\omega, \vec{k}}^{\dagger}$. These modes allow

\footnotetext{
${ }^{8}$ See the book [37] for a physics introduction, the summary done in ref. [26], or ref. [38] for a recent review.
} 
for the evaluation of the series of nested commutators (2.1) in any energy eigenstate compatible with the given temperature if the theory satisfies the ETH conjecture. Within the large-N limit, ${ }^{9}$ this operator structure should be enough to characterize any notion of operator growth.

In this section, we confirm this last expectation by exploring several notions of operator growth. We start by reviewing some recently introduced notions of operator growth, such as operator size in spin systems or the recursion method in many-body physics. We then provide a general framework to study operator growth in QFT, based on the GNS construction reviewed in appendix A. Lastly we apply the approach to large- $\mathrm{N}$ theories. Albeit our applications will be confined to such theories, the present GNS approach might help to understand the putative definitions of operator growth in generic QFT's.

We close the section with a discussion on the relation between Nielsen's geometric formulation of quantum circuit complexity, operator growth and quantum/classical chaos. In particular, we show that the quantum circuit complexity of a perturbed state equals the cost of generating the time evolved operator responsible for the perturbation. Quantum circuit complexity and operator growth are thus functionally dependent in this precise manner. Using the results in previous sections, we conclude both are determined by the nested operators (2.1) we computed in the large- $\mathrm{N}$ limit.

\subsection{Operator growth as state mixing in the GNS construction}

To define any notion of operator growth, it is natural to require the ability to expand a given operator in different bases of the space of operators, in order to quantify how the support of the operator changes with time. Hence, given an operator algebra $\mathcal{A}$, we need an inner product endowing $\mathcal{A}$ with the structure of a Hilbert space. This is precisely the goal of the GNS construction, which is reviewed in appendix A. Here we briefly summarize its main ingredients. Given a state ${ }^{10} \phi$ acting on the algebra $\mathcal{A}$ satisfying

$$
A \in \mathcal{A}, \quad \phi\left(A^{\dagger} A\right)=0 \quad \Longleftrightarrow \quad A=0,
$$

the GNS Hilbert space $\mathcal{H}_{\phi}$ and its inner product are defined by

$$
A \in \mathcal{A} \Rightarrow|A\rangle \in \mathcal{H}_{\phi}, \quad\langle B \mid A\rangle \equiv \phi\left(B^{\dagger} A\right) .
$$

In this Hilbert space there are two equivalent representations $\pi$ and $\bar{\pi}$ of the algebra $\mathcal{A}$ acting on $\mathcal{H}_{\phi}$

$$
A \in \mathcal{A} \Rightarrow|A\rangle \in \mathcal{H}_{\phi}, \quad \pi(A)|B\rangle \equiv|A B\rangle, \quad \bar{\pi}(A)|B\rangle \equiv\left|B A^{\dagger}\right\rangle .
$$

\footnotetext{
${ }^{9}$ Our discussion focuses on large- $\mathrm{N}$ theories, but it also applies to free QFT in Rindler space given the algebraic equivalence between Rindler operators and the Fourier modes $\mathcal{O}_{\omega, \vec{k}}$ and $\mathcal{O}_{\omega, \vec{k}}^{\dagger}$, as explicitly discussed in section 3. To our knowledge, operator growth in Rindler space has not been considered in the literature and it is useful to gauge away some of the confusions arising when defining the notion of operator growth in QFT.

${ }^{10}$ The word state refers to a linear functional acting on the algebra $\mathcal{A}$ as properly defined in (A.2). This is the standard terminology used in algebraic QFT [37].
} 
This construction is valid for any type of operator algebras, including the type III algebras relevant for QFT.

Consider states $|\kappa\rangle$ arising from abstract states in the algebra which are invariant under time evolution, such as thermofield double states. ${ }^{11}$ In this context, the GNS construction maps the Heisenberg time evolution $A(t)$ of any operator belonging to the algebra to the Schrödinger's time evolution of the associated GNS state

$$
U(t) \pi(A)|\kappa\rangle \equiv \pi(A(t))|\kappa\rangle=|A(t) \kappa\rangle \equiv|\Psi(t)\rangle,
$$

The same conclusion holds for the representation $\bar{\pi}$.

Since operator evolution is equivalent to state evolution in the associated GNS Hilbert space, any notion of operator growth should be characterized by expectation values of size operators in the GNS Hilbert space $\mathcal{H}_{\phi}$

$$
\left\langle\Psi(t)\left|\sum_{i j} \bar{\pi}\left(B_{i}\right) \pi\left(B_{j}\right)\right| \Psi(t)\right\rangle
$$

as any other property attached to states in $\mathcal{H}_{\phi}$. In the previous relation $B_{j}$ runs over a basis of operators of the algebra.

Albeit the previous formulation might seem too abstract, below we describe how recent examples in lattice systems look through this light. The advantage of the present formulation is that it will prove to be useful and trasnparent as well when we move towards large-N CFT's.

Operator size for simple Majorana operators. Before exploring this perspective for large- $\mathrm{N}$ theories, we briefly comment on how the case of Majorana spin systems at infinite temperature [1] and its extension to finite temperature [2] fit in this framework.

Consider a set of $N$ fundamental Majorana operators normalized by $\left\{\psi_{a}, \psi_{b}\right\}^{2}=2 \delta_{a b}$. Every operator $\psi$ in the algebra $\mathcal{A}$ can be expanded as

$$
\mathcal{O}=\sum_{s=1}^{N} \sum_{a_{1} \cdots a_{s}} c_{a_{1} \cdots a_{s}} \psi_{a_{1}} \cdots \psi_{a_{s}} .
$$

The size of such operator was defined by [1]

$$
S_{\mathcal{O}}=\sum_{s=1}^{N} s \sum_{a_{1} \cdots a_{s}}\left|c_{a_{1} \cdots a_{s}}\right|^{2} .
$$

This is natural if one thinks of the label $s$ as describing, either location in a 1d lattice, or directly in terms of the number of fundamental fermions building the operator.

The connection to the GNS construction is as follows. One assigns a vector $|\mathcal{O}\rangle \in \mathcal{H}_{\phi}$ to every operator $\mathcal{O} \in \mathcal{A}$ with GNS inner product

$$
\left\langle\mathcal{O} \mid \mathcal{O}^{\prime}\right\rangle \equiv \frac{1}{Z} \operatorname{Tr}\left(\mathcal{O}^{\dagger} \mathcal{O}^{\prime}\right)
$$

\footnotetext{
${ }^{11}$ Notice that this can be straightforwardly generalized to states invariant under so-called modular time evolution. Also, notice that this definition is not restricted to time-independent states. Starting with the invariant one we can move to other states by using elements of the algebra. These states would then evolve as it is described.
} 
where $Z$ is the dimension of the Hilbert space $Z=\operatorname{Tr}(\mathbb{1})$ induced by the normalized inner product for finite matrices. It follows the identity element of the algebra $\mathbb{1} \rightarrow|\mathbb{1}\rangle$ and the inner product can be interpreted as an expectation value at infinite temperature. There is one natural representation of the algebra in $\mathcal{H}_{\phi}$, defined by $\pi(\mathcal{O})\left|\mathcal{O}^{\prime}\right\rangle=\left|\mathcal{O O}^{\prime}\right\rangle$. This allows to define unitary time evolution in the GNS Hilbert space by

$$
U_{t} \pi(\mathcal{O})|\mathbb{1}\rangle \equiv \pi(\mathcal{O}(t))|\mathbb{1}\rangle=|\mathcal{O}(t)\rangle .
$$

This gives a concrete example on how operator evolution is seen as state evolution in the GNS Hilbert space.

Within the GNS construction, there exists an operator $\hat{S}$ acting on $\mathcal{H}_{\phi}$

$$
\hat{S}=\sum_{s=1}^{N} s \sum_{a_{1} \cdots a_{s}} \pi\left(\psi_{a_{1}} \cdots \psi_{a_{s}}\right)|\mathbb{1}\rangle\left\langle\mathbb{1}\left|\pi\left(\psi_{a_{1}} \cdots \psi_{a_{s}}\right)=\sum_{s=1}^{N} s \sum_{a_{1} \cdots a_{s}}\right| \psi_{a_{1}} \cdots \psi_{a_{s}}\right\rangle\left\langle\psi_{a_{1}} \cdots \psi_{a_{s}}\right|,
$$

satisfying

$$
S_{\mathcal{O}}(t)=\langle\mathcal{O}(t)|\hat{S}| \mathcal{O}(t)\rangle
$$

Hence, the notion of size (4.7) equals the expectation value of $\hat{S}$ in the GNS state $|\mathcal{O}(t)\rangle$ that is mapped to the time evolution of the original operator $\mathcal{O}(t)$. This matches our general expectation that any notion of operator growth should be computable by expectation values evaluated on the GNS state. In fact, introducing creation $c_{i}^{\dagger}$ and annihilation $c_{i}$ operators in $\mathcal{H}_{\phi}$ by

$$
\begin{aligned}
c_{i}\left|\psi_{a_{1}} \cdots \psi_{a_{s}}\right\rangle & =\delta_{i, a_{1}}\left|\psi_{a_{2}} \cdots \psi_{a_{s}}\right\rangle+\cdots+\delta_{i, a_{s}}\left|\psi_{a_{2}} \cdots \psi_{a_{s-1}}\right\rangle, \\
c_{i}^{\dagger} c_{i}\left|\psi_{a_{1}} \cdots \psi_{a_{s}}\right\rangle & =\delta_{i, a_{1}} \cdots \delta_{i, a_{s}},
\end{aligned}
$$

the size operator (4.10) can be reinterpreted as a number operator

$$
\hat{S}=\sum_{i=1}^{N} c_{i}^{\dagger} c_{i}
$$

Hence, we learn there is a basis of operators in $\mathcal{H}_{\phi}$ where a natural notion of size in this lattice system is a simple quadratic operator.

This notion of size in lattice systems was extended to finite temperature in [2] by purifying the thermal ensemble of the Majorana fermions. Such an approach is directly of the GNS form, as reviewed in appendix A.1, but using fermionic operators. Hence, our conclusions extend to this finite temperature case too.

Alternative notions of operator size Depending on the dynamics and state of the system, operator size defined as in (4.7) may not be a dynamical quantity. This can happen even if the complexity of the operator grows. Indeed, if the Hamiltonian preserves the number of particles, the previous definition of operator size will be a conserved charge for a natural class of initial states, as we review now.

Consider a bunch of spinless fermions whose hamiltonian conserves the number of particles. Any state can be expanded as

$$
|\psi\rangle=\sum_{s=0}^{N} \sum_{a_{1} \cdots a_{s}} \psi_{a_{1} \cdots a_{s}} c_{a_{1}}^{\dagger} \cdots c_{a_{s}}^{\dagger}\left|\downarrow_{1} \cdots \downarrow_{N}\right\rangle,
$$


where $c_{i}^{\dagger}$ and $c_{i}$ create and destroy such fermions at site $i$. Besides operator size, one can ask about how many particles are being transported by the Hamiltonian as time evolves. This was considered in [46]. To be definite, consider an starting state with the first $m$ particles excited. Unitary evolution mixes the state with other states in the $m$-particle sector

$$
|\psi(t)\rangle=\sum_{a_{1} \cdots a_{m}} \psi_{a_{1} \cdots a_{m}}(t) c_{a_{1}}^{\dagger} \cdots c_{a_{m}}^{\dagger}\left|\downarrow_{1} \cdots \downarrow_{N}\right\rangle .
$$

One proposed notion of operator growth is the average number of jumps (the average transport) the spins have performed due to Hamiltonian evolution. Such number can be measured by the expectation value of the number operator

$$
\hat{T} \equiv \sum_{i=m+1}^{N} c_{i}^{\dagger} c_{i}
$$

counting the number of fermions in the sites that were not populated at $t=0$. It follows

$$
T(t) \equiv\langle\psi(t)|\hat{T}| \psi(t)\rangle
$$

is the average particle transport. This is a sensible measure of the growth of the state $|\psi(t)\rangle$ and it is still the expectation value of a simple operator. Also, since

$$
|\psi(t)\rangle=U(t) c_{1}^{\dagger} \cdots c_{m}^{\dagger} U^{-1}(t)\left|\downarrow_{1} \cdots \downarrow_{N}\right\rangle \equiv \mathcal{O}(t)\left|\downarrow_{1} \cdots \downarrow_{N}\right\rangle,
$$

this expectation value is equivalently studying the growth of the operator $\mathcal{O}=c_{1}^{\dagger} \cdots c_{m}^{\dagger}$ in the state $\left|\downarrow_{1} \cdots \downarrow_{N}\right\rangle$. Notice that while this notion of size is bound to grow, as analized in [46], the previous notion of size, where we would add up all spins in the definition (4.16), would be constant through time evolution due to particle number conservation.

\subsection{Size, number operators and energies in large- $N$ and holographic theories}

The observation that Heisenberg operator evolution is equivalent to Schrödinger's time evolution in the GNS Hilbert space provides a hint to extend the notion of operator size to QFT. Such notion is based on simple operators, such as (4.13) and (4.16) in spin systems (see $[1,2,13,46])$.

Given the structure of time evolution for holographic CFTs in the large-N limit discussed in sections 2 and 3.2, it may be natural to define any notion of operator size as being of the form

$$
\hat{S}=\sum_{\alpha} F_{\alpha}\left[\left(\mathcal{O}_{\alpha}\right)_{\omega, \vec{k}},\left(\mathcal{O}_{\alpha}^{\dagger}\right)_{\omega, \vec{k}},\left(\tilde{\mathcal{O}}_{\alpha}\right)_{\omega, \vec{k}},\left(\tilde{\mathcal{O}}_{\alpha}^{\dagger}\right)_{\omega, \vec{k}}\right]
$$

in terms of the operator modes and its mirror partners for the different local low conformal dimension boundary operators indexed by $\alpha$. The additive nature on the spectrum of operators is due to the absence of mixing between operators when neglecting $1 / \mathrm{N}$ corrections.

Assuming the generic definition (4.19), large- $\mathrm{N}$ factorization ensures that any notion of size in large- $\mathrm{N}$ theories associated with a choice of the functionals $F_{\alpha}$ is completely 
determined by the two-point function (2.6), up to $1 / \mathrm{N}$ corrections. Indeed, to study the growth of the size of a certain field $\mathcal{O}(t)$ in the thermofield double $\left|\kappa_{\beta}\right\rangle$ at temperature $\beta$, we just need to take the expectation value of $\hat{S}$ in the evolving GNS state $\left|\mathcal{O}(t) \kappa_{\beta}\right\rangle$. Such expectation value can be computed by using expressions (2.2) and (2.6), together with large- $\mathrm{N}$ factorization.

Let us remark that in previous literature, starting with [1], the notion of size has been argued to be related to out-of-time ordered correlation functions. If this relation is extended to any large- $\mathrm{N}$ theory and any temperature, our analysis would imply a nontrivial relation between two and four-point functions in the large- $\mathrm{N}$ limit. These aspects will be studied elsewhere.

In analogy to the spin size (4.13), natural choices for the size $\hat{S}$ in large-N QFTs are simple operators like the number or energy operators associated to the different bases (Rindler, Unruh, and Minkowski) of creation and annihilation operators discussed in section 3.2. As in our discussion of the dynamics preserving the number of particles, not all choices for such operators will provide useful dynamical information. In what follows, the upper index in the size operator refers to the basis chosen, either Rindler (R), Unruh (U) or Minkowski (M), and the lower index to whether it is energy-based $(\mathrm{H})$ or particle number based $(\mathrm{N})$.

Let us start our discussion with the number and energy operator associated to the standard basis of operator modes

$$
\hat{S}_{\mathrm{N}}^{\mathrm{R}}=\hat{N}=\int_{\omega>0} d \omega d^{d-1} \vec{k} \mathcal{O}_{\omega, \vec{k}}^{\dagger} \mathcal{O}_{\omega, \vec{k}} \quad \text { and } \quad \hat{S}_{\mathrm{H}}^{\mathrm{R}}=\hat{H}=\int_{\omega>0} d \omega d^{d-1} \vec{k} \omega \mathcal{O}_{\omega, \vec{k}}^{\dagger} \mathcal{O}_{\omega, \vec{k}}
$$

Then the expectation value $\left\langle\kappa_{\beta} \mathcal{O}(t)|\hat{S}| \mathcal{O}(t) \kappa_{\beta}\right\rangle$ is constant for any operator $\mathcal{O}$ and provides no further dynamical information.

Unruh and Minkowski number operators. Consider the Unruh and Minkowski number operators choice

$$
\hat{S}_{\mathrm{N}}^{\mathrm{U}}=\hat{N}^{\mathrm{U}}=\int d k_{z} d^{d-1} \vec{k} \mathcal{O}_{\omega \vec{k}}^{\mathrm{U} \dagger} \mathcal{O}_{\omega \vec{k}}^{\mathrm{U}}, \quad \text { and } \quad \hat{S}_{\mathrm{N}}^{\mathrm{M}}=\hat{N}^{\mathrm{M}}=\int d k_{z} d^{d-1} \vec{k} \mathcal{O}_{k_{z} \vec{k}}^{\mathrm{M} \dagger} \mathcal{O}_{k_{z} \vec{k}}^{\mathrm{M}} .
$$

Both choices are equal due to the algebra of field modes. This proposal is inspired by the relation (3.22), which is a specific instance of the more general Tomita-Takesaki like equation (A.18). One basically defines the simplest operators annihilating the thermofield double $^{12}$ and uses them to define a number operator. For Majorana fermions this was the path chosen in [2]. Here we see that such operators, in the black hole scenario, are the known Unruh creation/annihilation operators.

These notions of size give zero on the thermofield double, while positive sizes on the thermofield double with perturbations. However, if we consider a Minkowski mode excita-

\footnotetext{
${ }^{12}$ Notice there is an infinite number of choices that also annihilate the thermofield double, including the Minkowski annihilation/creation operators.
} 
tion generated by $\mathcal{O}_{k_{z} \vec{k}}^{\mathrm{M} \dagger},{ }^{13}$ this notion of size will remain constant through time evolution. This is because unitary evolution acts like a boost on these excitations. Hence, it changes the value of their momentum but not the number of modes. This choice shows that depending on the basis of operators being considered, equating size with number operators may not provide useful dynamical information.

Minkowski energy. Consider the boundary CFT operator describing the analog of a bulk infalling Hamiltonian ${ }^{14}$ constructed in the previous section

$$
\hat{S}_{\mathrm{H}}^{\mathrm{M}}=H^{\mathrm{M}}=\int d k_{z} d^{d-1} \vec{k} \omega_{\vec{k}} \mathcal{O}_{k_{z} \vec{k}}^{\mathrm{M} \dagger} \mathcal{O}_{k_{z} \vec{k}}^{\mathrm{M} \dagger}
$$

This choice was studied in detail in [10]. Since the CFT time evolution acts as a boost upon a Minkowski mode $\mathcal{O}_{k_{z} \vec{k}}^{\mathrm{M} \dagger}$

$$
e^{i \gamma K} \mathcal{O}_{k_{z} \vec{k}}^{\mathrm{M} \dagger} e^{-i \gamma K}=\frac{\sqrt{\gamma\left(\omega_{\vec{k}}\right)}}{\sqrt{\omega_{\vec{k}}}} \mathcal{O}_{\gamma\left(k_{z}\right) \vec{k}}^{\mathrm{M} \dagger},
$$

it follows the size will exponentially grow at large times $\left(t^{\mathrm{M}} \gg \beta\right)$, with Lyapunov exponent equal to $2 \pi / \beta$, since evolving with the QFT Hamiltonian for time $t$ is equivalent to boosting the particle with rapidity $\frac{2 \pi}{\beta} t$ (see (3.29)). This shows that the usual exponential blueshift of the proper energy $E_{\mathrm{p}} \sim e^{\frac{2 \pi}{\beta} t}$ of infalling observers (perturbations) in black hole physics, which simply rests on the universal structure of the time component of the metric $\left(g_{00}\right)$ near the horizon, can be seen as measuring the growth of the size of the associated perturbation in the CFT.

\subsection{The recursion method at large- $\mathrm{N}$}

A standard approach in condensed matter physics to study the Heisenberg time evolution and complexity of operators is the recursion method (see [18] for a detailed presentation). This perspective on operator growth was recently considered in SYK in [13] and used to study long time scales of operator dynamics in [14]. In this section we explore what the structure of time evolution in large- $\mathrm{N}$ theories described in sections 2 and 3.2 teaches us about this approach.

Let us first describe this method briefly. As before, the recursion method requires the definition of an inner product. The book [18] considers a whole family of them, but we show in appendix A.2 that all choices can be related to one convenient representative in QFT. In the following we focus on such representative and, for simplicity, we only

\footnotetext{
${ }^{13}$ We remark that although the Minkowski creation/annihilation operators are state-dependent in the one-sided case since they make use of the mirror operators, exciting a Minkowski mode is not a statedependent action. The reason is that the action of the mirror creation operator can be fully mimicked by their partners in the right wedge, as in (3.11).

${ }^{14}$ Here we are referring to the Minkowski energy choice. The Unruh energy is not a sensible choice since it is infinite for any Minkowski mode.
} 
consider operators with vanishing one-point functions. Given two operators $A$ and $B$, the representative inner product $(A, B)$ is defined by

$$
(A, B) \equiv\left\langle e^{\beta H / 2} A^{\dagger} e^{-\beta H / 2} B\right\rangle_{\beta},
$$

where $\langle A\rangle_{\beta}=\operatorname{Tr}\left(\rho_{\beta} A\right)$. Within the GNS framework, this inner product can be written as

$$
\langle\kappa|\bar{\pi}(A) \pi(B)| \kappa\rangle=\left\langle\kappa \mid B \kappa A^{\dagger}\right\rangle=\left\langle e^{\beta H / 2} A^{\dagger} e^{-\beta H / 2} B\right\rangle_{\beta}=(A, B) .
$$

The inner product (4.24) allows to expand any hermitian operator $\mathcal{O}(t)$ in an orthogonal basis of operators

$$
\mathcal{O}(t)=\sum_{k=0}^{\infty} C_{k}(t) f_{k}, \quad \text { with } \quad\left(f_{k}, f_{k^{\prime}}\right)=\left(f_{k}, f_{k}\right) \delta_{k k^{\prime}}
$$

for some time dependent coefficients $C_{k}(t)$. The recursion method proposes to use an explicit basis $f_{k}$, the Lanczos basis, to study operator evolution. Basically, starting from the operators $\mathcal{O}_{n}$ defined previously, it provides a constructive algorithm, based on the Gram-Schmidt orthogonalization procedure, to determine such basis

$$
f_{k+1}=i\left[H, f_{k}\right]+\Delta_{k} f_{k-1}, \quad k=0,1, \ldots \quad \text { with } \quad \Delta_{k}=\frac{\left(f_{j}, f_{j}\right)}{\left(f_{j-1}, f_{j-1}\right)}, j=1,2, \ldots
$$

with initial conditions $f_{-1}=0$ and $f_{0}=\mathcal{O}$ being the operator at initial time. The coefficients $\Delta_{k}$ are referred to as the Lanczos coefficients. The linear operator $\mathcal{L}$ generating the time evolution as $\mathcal{L}(\mathcal{O}) \equiv[H, \mathcal{O}]$ is sometimes called the Liouvillian. It corresponds to the GNS Hamiltonian in the GNS construction described in appendix A generating the unitary evolution in (A.14)-(A.15).

Plugging the expansion (4.26) into Heisenberg's equation of motion, one derives a $1 \mathrm{~d}$ diffusion equation for the amplitudes $C_{k}(t)$

$$
\frac{d \mathcal{O}}{d t}=i \mathcal{L} \mathcal{O} \quad \Leftrightarrow \quad \dot{C}_{k}(t)=C_{k-1}(t)-\Delta_{k+1} C_{k+1}(t), \quad k=0,1,2 \ldots
$$

with initial conditions $C_{-1}(t)=0$ and $C_{k}(0)=\delta_{k 0}$. In this framework, the Lanczos operator complexity $L_{\mathcal{O}}$ of an operator $\mathcal{O}$ can be defined by the average position in this effective $1 \mathrm{~d}$ chain $^{15}$

$$
L_{\mathcal{O}} \equiv \sum_{k=0}^{\infty} k \frac{\left|\left(f_{k}, \mathcal{O}(t)\right)\right|^{2}}{\left(f_{k}, f_{k}\right)} .
$$

\footnotetext{
${ }^{15}$ When adopting this definition, one is assigning some kind of locality interpretation to the $1 \mathrm{~d}$ chain which was manifest in our Majorana fermion discussion (4.7). Here, the label $k$ technically accounts for the number of commutators with $H$ that have acted upon the starting operator. Thus, depending on the interactions in this hamiltonian, the support of the different $f_{k}$ operators will grow accordingly. Since one is interested in quantifying the evolution in the size of this support, one could consider more general functionals $\sum_{k=0}^{\infty} g(k) \frac{\left|\left(f_{k}, \mathcal{O}(t)\right)\right|^{2}}{\left(f_{k}, f_{k}\right)}$ capturing this quantity more precisely. Our point here is that the existent inner product allows to define a notion of average size for any relevant choice of $g(k)$.
} 
Because of (4.25), $L_{\mathcal{O}}$ equals the expectation value of the "Lanczos operator" in the GNS Hilbert space

$$
\hat{L}_{\mathcal{O}} \equiv \sum_{k=0}^{\infty} k \frac{\bar{\pi}\left(f_{k}\right)}{\sqrt{\left(f_{k}, f_{k}\right)}}|\kappa\rangle\langle\kappa| \frac{\bar{\pi}\left(f_{k}\right)}{\sqrt{\left(f_{k}, f_{k}\right)}}
$$

so that

$$
L_{\mathcal{O}}=\left\langle\kappa\left|\pi(\mathcal{O}(t)) \hat{L}_{\mathcal{O}} \pi(\mathcal{O}(t))\right| \kappa\right\rangle .
$$

Notice $\hat{L}_{\mathcal{O}}$ is a state dependent operator in the GNS Hilbert space, i.e. it is not linear in $\mathcal{O}$.

Knowledge of the Lanczos coefficients $\Delta_{n}$ determines the orthogonal basis $\left\{f_{k}\right\}$ and allows to determine the full-time evolution of the operator $\mathcal{O}(t)$ through the integration of the $1 \mathrm{~d}$ diffusion equation (4.28). Interestingly, these coefficients $\Delta_{n}$ can be recursively extracted from the connected 2-pt function

$$
Q(t) \equiv\langle\mathcal{O}(t) \mathcal{O}(0)\rangle_{\beta}
$$

This can be seen as follows [18]. Since

$$
C_{0}(t) \equiv \frac{(\mathcal{O}(t), \mathcal{O})}{(\mathcal{O}, \mathcal{O})}=\frac{\Phi(t)}{\Phi(0)}
$$

where $\Phi(t) \equiv(Q(t)+Q(-t)) / 2$, if one assumes the Taylor expansion

$$
C_{0}(t)=\sum_{k=0}^{\infty} \frac{(-1)^{k}}{(2 k) !} M_{2 k} t^{2 k}
$$

is sensible, it follows the existence of a one-to-one reconstruction algorithm between the moments $M_{2 k}$, which are determined solely by derivatives of the 2-pt function (4.32), and the Lanczos coefficients $\Delta_{k}$

$$
M_{2 k}^{(n)}=\frac{M_{2 k}^{(n-1)}}{\Delta_{n-1}}-\frac{M_{2 k-2}^{(n-2)}}{\Delta_{n-2}}, \quad \Delta_{n}=M_{2 n}^{(n)}, \quad k=n, n+1, \ldots, K \quad \text { and } n=1,2, \ldots, K
$$

The initial conditions of this recursion are $M_{2 k}^{(0)}=M_{2 k}$ and $\Delta_{-1}=\Delta_{0}=1$ and $M_{2 k}^{(-1)}=0$.

In section 2 , the structure of time evolution in large- $\mathrm{N}$ theories was discussed. It is natural to ask whether we can learn anything about the recursion method given this structure. The first observation is that the operators $\mathcal{O}_{\omega, \vec{k}}$ diagonalize the Liouvillian

$$
\frac{d \mathcal{O}_{\omega, \vec{k}}(t)}{d t}=i \mathcal{L}\left(\mathcal{O}_{\omega, \vec{k}}\right)=i\left[H, \mathcal{O}_{\omega, \vec{k}}\right]=-i \omega \mathcal{O}_{\omega, \vec{k}} \quad \Longrightarrow \quad \mathcal{O}_{\omega, \vec{k}}(t)=e^{-i \omega t} \mathcal{O}_{\omega, \vec{k}}(t)
$$

Hence, there are two natural bases in the space of operators: the Lanczos basis, made of the orthogonal $f_{k}$ and the operator modes $\mathcal{O}_{\omega, \vec{k}}$ diagonalising the Liouvillian in the large-N limit. Finding the change of basis would immediately solve the diffusion equation (4.28), since the time evolution of the $\mathcal{O}_{\omega, \vec{k}}$ operators is known. Since properly normalised operator modes (3.19) satisfy the canonical commutation relation associated to a set of creation and annihilation operators, we analyse the latter from the recursion method perspective next. 
Consider $K$ free harmonic oscillators, denoted by $a_{j}$ and $a_{j}^{\dagger}$, with $j=1, \ldots, K$. Take as our starting operator in the recursion method algorithm

$$
f_{0}=\sum_{j} D_{j}\left(a_{j}+a_{j}^{\dagger}\right), \quad D_{i} \in \mathbb{R}
$$

with $D_{j}$ any set of coefficients. This choice matches the $t=0$ expansion of the field in (2.2) and accommodates their normalisation (3.19). Since $\mathcal{L} a_{j}^{\dagger}=\omega_{j} a_{j}^{\dagger}$ and $\mathcal{L} a_{j}=-\omega_{j} a_{j}$, it follows the vectors generated by the recursion method algorithm must be of the form

$$
\begin{aligned}
f_{2 k} & =\sum_{j} D_{j} P_{k, j}\left(a_{j}+a_{j}^{\dagger}\right), k=0,1, \ldots K-1 & \text { with } & P_{0, j}=1 \forall j \\
f_{2 k+1} & =\sum_{j} D_{j} Q_{k, j} i\left(a_{j}^{\dagger}-a_{j}\right), k=0,1, \ldots K-1 & \text { with } & Q_{0, j}=\omega_{j} \forall j
\end{aligned}
$$

for some unknown real coefficients $P_{k, j}$ and $Q_{k, j}$ satisfying the above initial conditions. Using the harmonic oscillator thermal correlators

$$
\left\langle a_{i} a_{j}^{\dagger}\right\rangle_{\beta}=e^{\beta \omega_{i}} n_{\beta}\left(\omega_{i}\right) \delta_{i j}, \quad\left\langle a_{i}^{\dagger} a_{j}\right\rangle_{\beta}=n_{\beta}\left(\omega_{i}\right) \delta_{i j},
$$

where $n_{\beta}(\omega)=\left(e^{\beta \omega}-1\right)^{-1}$, it follows

$$
\left(a_{j}+a_{j}^{\dagger}, a_{k}+a_{k}^{\dagger}\right)=\left(i\left(a_{j}^{\dagger}-a_{j}\right), i\left(a_{k}^{\dagger}-a_{k}\right)\right)=\frac{2}{\beta} \int_{0}^{\beta} d \lambda e^{\lambda \omega_{j}} n_{\beta}\left(\omega_{j}\right) \delta_{j k} \equiv A_{j} \delta_{j k},
$$

with all other inner product combinations vanishing. Hence, the set of vectors $\left\{f_{2 k}, f_{2 k+1}\right\}$ defines an orthogonal set, as it should. For $K$ finite oscillators, the algorithm will halt once we reach a basis of $2 K$ orthogonal vectors, which matches the number of independent creation/annihilation operators. This explicitly confirms the relation between the two set of operators is indeed simply a change of basis. This change is only non-trivial for $K>1$, as in large-N QFTs where there is an infinite set of operator modes when studying the growth of a local field.

Plugging the parameterisation (4.38) into (4.27), we obtain the following recurrence relations

$$
\begin{aligned}
P_{s, j} & =-\omega_{j} Q_{s-1, j}+\Delta_{2 s-1} P_{s-1, j}, & & s=1,2, \ldots K-1 \\
Q_{s, j} & =\omega_{j} P_{s, j}+\Delta_{2 s} Q_{s-1, j}, & s & =1,2, \ldots K-1
\end{aligned}
$$

These are solved by ${ }^{16}$

$$
\begin{aligned}
P_{s, j} & =\sum_{m=0}^{s}(-1)^{m} \omega_{j}^{2 m} \sum_{i_{1}=1}^{2 m+1} \Delta_{i_{1}} \sum_{i_{2}=i_{1}+2}^{2 m+3} \Delta_{i_{2}} \ldots \sum_{i_{s-m}=i_{s-m-1}+2}^{2 s-1} \Delta_{i_{s-m}}, \\
Q_{s, j} & =\omega_{j} \sum_{m=0}^{s}(-1)^{m} \omega_{j}^{2 m} \sum_{i_{1}=1}^{2(m+1)} \Delta_{i_{1}} \sum_{i_{2}=i_{1}+2}^{2(m+2)} \Delta_{i_{2}} \ldots \sum_{i_{s-m}=i_{s-m-1}+2}^{2 s} \Delta_{i_{s-m}} .
\end{aligned}
$$

The proof is by induction and it is given in appendix B.

\footnotetext{
${ }^{16}$ It is understood that whenever the subindex labels $r$ in $i_{r}$ equal zero, such terms do not contribute to the solution.
} 
As a check of our formal solution to the recursion method, we show it satisfies the diffusion equation (4.28). As stressed above, the advantage of expressing our operators in the basis of field modes is that these modes diagonalize the Liouvillian operator $\mathcal{L}$ and therefore make the time dependence trivial. Hence, the exact time evolution of our initial operator just equals

$$
f_{0}(t)=\sum_{j} D_{j}\left(e^{i t \omega_{j}} a_{j}^{\dagger}+e^{-i t \omega_{j}} a_{j}\right) .
$$

From this expression, to find the Lanczos expansion we just need to write the $a_{j}^{\dagger}$ and $a_{j}$ in terms of the $f_{k}$. We remark this is not a dynamical question, just a change of basis. To invert the relation we use that the amplitudes entering the diffusion equation are given by the projections

$$
C_{2 k}(t) \equiv \frac{\left(f_{2 k}, f_{0}(t)\right)}{\left(f_{2 k}, f_{2 k}\right)}, \quad C_{2 k+1}(t) \equiv \frac{\left(f_{2 k+1}, f_{0}(t)\right)}{\left(f_{2 k+1}, f_{2 k+1}\right)} .
$$

Explicit calculation yields

$$
\begin{aligned}
C_{2 k}(t) & =\sum_{j} D_{j}^{2} A_{j} \frac{P_{k, j}}{\left(f_{2 k}, f_{2 k}\right)} \cos \omega_{j} t, \\
C_{2 k+1}(t) & =\sum_{j} D_{j}^{2} A_{j} \frac{Q_{k, j}}{\left(f_{2 k+1}, f_{2 k+1}\right)} \sin \omega_{j} t .
\end{aligned}
$$

Computing the time derivatives and using the recursion relations (4.41) to replace the $\omega_{j}$ dependent terms reproduces the diffusion equation (4.28).

To sum up, the 2-pt function (4.32) determines the Lanczos coefficients $\Delta_{k}$ and these determine the orthonormal basis of operators $f_{k}$ controlling the Heisenberg time evolution of an initial operator $\mathcal{O}$. These are related by a change of basis to the operators used in section 2 to describe this same evolution in large- $\mathrm{N}$ gauge theories. This is consistent with our claim that 2-pt functions characterize operator growth in these theories at leading order. In this approximation, what makes these theories special, from the recursion method perspective, is that we can solve the diffusion equation analytically by relations (4.45), once we have the coefficients $\Delta_{k}$. In QFT, the set of modes is infinite and the recursion does not halt. The operator then grows indefinitely, even if we are dealing with a set of free harmonic oscillators.

The specific structure of the Lanczos basis unraveled here also differs from the general exponential growth of the Lanczos complexity in QFT. As remarked in [13], in the typical QFT scenario in which the 2-pt function is exponentially decaying and its Fourier transform has poles, as dictated by the generic analyticity properties of thermal correlations, then the mean position in the one-dimensional diffusion equation (4.28), the Lanczos operator complexity (4.29), will grow exponentially fast with Lyapunov exponent $2 \pi / \beta .{ }^{17}$ Such statement holds for any chaotic QFT, and it is not particular to large-N theories.

\footnotetext{
${ }^{17}$ As shown in appendix A.2, this exponential growth with the right Lyapunov exponent only applies to the representative inner product considered in (4.24). For other inner products, one gets exponential growths with faster rates. This statement seems similar to the results found in [47] for out-of-time-ordered correlation functions, which show that different choices of euclidean separations in the OTOC 4-pt function might lead to faster growth than the chaos bound [8].
} 


\subsection{Chaos and quantum complexity}

In this section we first stress the existence of a natural relation between operator growth and quantum circuit complexity. Afterwards, we discuss the link between these two concepts and quantum chaos $[10,12,15]$. Finally, in the context of the AdS/CFT correspondence, we also briefly comment on the relation between them and the emergence of classical bulk chaos.

In quantum complexity discussions, the complexity $\mathcal{C}_{|\psi\rangle}$ to prepare a particular target state $|\psi\rangle$ starting with a certain reference state $\left|\psi_{\mathrm{R}}\right\rangle$ by applying a series of elementary gates $g_{i}$

$$
|\psi\rangle=U_{\mathrm{R}}\left|\psi_{\mathrm{R}}\right\rangle=g_{n} \cdots g_{2} g_{1}\left|\psi_{\mathrm{R}}\right\rangle
$$

is defined as the number of gates associated to the optimal protocol. Nielsen and collaborators $[16,17,48]$ mapped the problem of identifying this optimal circuit to the geometric problem of finding a geodesic in the space of unitaries acting on the Hilbert space. Given a one parameter family of states, labelled by $s$, the local driving hamiltonian $H(s)$ satisfies

$$
i \frac{d}{d s}|\psi(s)\rangle=H(s)|\psi(s)\rangle
$$

and generates the unitary transformation acting on the state

$$
U(\sigma)=\stackrel{\mathfrak{P}}{\exp }\left[-i \int_{0}^{\sigma} d s H(s)\right], \quad \text { with } \quad H(s) \equiv \sum_{\mathrm{I}} Y^{\mathrm{I}}(s) \mathcal{O}_{\mathrm{I}},
$$

where the Hermitian operators $\mathcal{O}_{I}$ generate the individual gates $g_{\mathrm{I}}$. Circuits satisfying eq. (4.46) correspond to trajectories satisfying the boundary conditions

$$
U(\sigma=0)=\mathbb{1}, \quad U(\sigma=1)=U_{\mathrm{R}} .
$$

Optimal circuits minimise the cost defined as

$$
\mathcal{C}_{\left|\psi_{\mathrm{R}}\right\rangle \rightarrow|\psi\rangle} \equiv \int_{0}^{1} d s F(H(s))
$$

where $F$ is a local cost function depending on the tangent vector $H(s)$.

Consider two states, $|\psi\rangle$, as above, and a perturbed state $\left|\psi_{\mathcal{O}}\right\rangle=e^{i \mathcal{O}}|\psi\rangle$, generated by the action of a simple unitary generated by certain local operator $\mathcal{O}$. The time evolution of both states is determined by the unitary action $U(t)=e^{-i H t}$ on them, where $H$ corresponds to the physical hamiltonian of the system, leading to the states $|\psi(t)\rangle$ and $\left|\psi_{\mathcal{O}}(t)\right\rangle$, respectively. In this set-up, one can define some notion of growth or size based on the circuit complexity to go from one evolved state to the other ${ }^{18}$

$$
S_{\mathcal{O}(t)} \equiv \mathcal{C}_{|\psi(t)\rangle \rightarrow\left|\psi_{\mathcal{O}}(t)\right\rangle}
$$

This relative complexity is simpler than expected in the limit of small perturbations. As shown in the original geometric complexity paper [16], if the perturbation is small

\footnotetext{
${ }^{18}$ This might be related to the complexity variation $\mathcal{C}_{|\psi(t)\rangle}-\mathcal{C}_{\left|\psi_{\mathcal{O}}(t)\right\rangle}$ considered in [12]. Variations in quantum circuit complexity have also been considered recently in [15, 49].
} 
enough so that $|\psi\rangle$ and $\left|\psi_{\mathcal{O}}\right\rangle$ are sufficiently closed to each other, the geodesic connecting them is simply

$$
U(s)_{|\psi\rangle \rightarrow\left|\psi_{\mathcal{O}}\right\rangle}=e^{i \mathcal{O} s}, \quad 0 \leqslant s \leqslant 1
$$

The key observation now is that the geodesic connecting the time evolved states $|\psi(t)\rangle$ and $\left|\psi_{\mathcal{O}(t)}\right\rangle$ is also going to be of the same type

$$
U(s)_{|\psi(t)\rangle \rightarrow\left|\psi_{\mathcal{O}}(t)\right\rangle}=e^{i \mathcal{O}(-t) s}, \quad 0 \leqslant s \leqslant 1
$$

by dialling the initial perturbation to be small enough. This argument allows to write the relative complexity of such geodesic as

$$
S_{\mathcal{O}(t)} \equiv \mathcal{C}_{|\psi(t)\rangle \rightarrow\left|\psi_{\mathcal{O}}(t)\right\rangle}=\int_{0}^{1} d s F(\mathcal{O}(-t))=F(\mathcal{O}(-t))
$$

The precise evaluation requires a choice of the cost function. See [15] for a discussion and calculation of several possibilities.

Connection to previous notions of size. Equation (4.54) states that the circuit complexity is the computational cost of the time evolved operator responsible for the perturbation. ${ }^{19}$ The final value depends on the choice of a cost function, pretty much as in our earlier discussions on operator size, the latter depends on the definition of size. Crucially, (4.54) stresses that, given some cost function, circuit complexity only depends on the time evolution of the operator, the same structure controlling any notion of operator growth or size. Hence, both notions are functionally dependent. In particular, if we were to define the cost as one of the previous notions of operator size, both would be equivalent. A convenient choice then is the Minkowski energy discussed in the previous section. With this choice, the relative complexity of large- $\mathrm{N}$ theories will grow exponentially fast with Lyapunov exponent $\lambda=2 \pi / \beta .^{20}$

Connection to chaos. Chaotic behavior concerns the sensitivity of certain dynamical systems to small perturbations $\delta x_{i}$ of its initial conditions. Classically, such sensitivity is usually studied in a double scaling limit, where the size of the perturbation is taken to zero first and the limit of large times is taken afterward. The first limit ensures that a linearized equation of the type

$$
\delta x_{i}(t)=\sum_{j} M_{i j} \delta x_{i}
$$

is a good approximation to the dynamics, where the Jacobian matrix $M_{i j}=\partial x_{i}(t) / \partial x_{j}$ encodes the dependence on the initial conditions $x_{j}$. The second limit ensures the solution to (4.55) is dominated by the largest Lyapunov exponent.

\footnotetext{
${ }^{19}$ The connection between cost functions and operator size was recognized already in [10] for spin systems, but equation (4.54) shows it holds more generally.

${ }^{20}$ This construction and the Minkowski energy choice for the complexity cost provides a specific realization of the idea put forward in [10], in which the cost function was argued to be related to the scaling dimension of the associated perturbation.
} 
A natural extension of the classical definition to the quantum domain has been recently developed in [15]. As shown in [50], quantum dynamics can be formulated as classical dynamics on a "quantum phase space", defined to be the Hilbert space itself. The symplectic form at point $|\psi\rangle$ along two infinitesimal directions, generated by Hamiltonian operators $H_{1}$ and $H_{2}$ is just the expectation value of the commutator $\Omega_{|\psi\rangle}\left(H_{1}, H_{2}\right) \equiv\left\langle\psi\left|\left[H_{1}, H_{2}\right]\right| \psi\right\rangle$. It turns out that Schrödinger equations are seen as Hamilton equations in such a phase space with "classical Hamiltonian" $H(|\psi\rangle)=\langle\psi|\hat{H}| \psi\rangle$. Having framed quantum dynamics as a classical system, it is most natural to define quantum chaos by the usual classical definition (4.55) but applied to the quantum phase space. This definition has, by construction, the appropriate pullback to the classical definition on a semiclassical phase space, but it is otherwise valid through the whole quantum system.

The classical approach to quantum mechanics illuminates the relation between operator growth and chaos by showing the transparent relation between $\mathcal{O}(-t)$, the operator that generates the unitary interpolating between the nearby quantum states at time $t$, and the Jacobian matrix associated to the classical chaotic process. In the Hilbert space we can define generalized coordinates $\left|q_{i}, p_{i}\right\rangle$ (at least locally) satisfying the canonical Poisson brackets with respect to the Hilbert space symplectic form. Generic infinitesimal perturbations of any state can be written as ${ }^{21}$

$$
e^{i \mathcal{O}}\left|q_{i}, p_{i}\right\rangle \equiv e^{i\left(\hat{p}_{i} \delta q_{i}-\hat{q}_{i} \delta p_{i}\right)}\left|q_{i}, p_{i}\right\rangle=\left|q_{i}+\delta q_{i}, p_{i}+\delta p_{i}\right\rangle \quad \Longrightarrow \quad \mathcal{O}=\sum_{i} \hat{p}_{i} \delta q_{i}-\hat{q}_{i} \delta p_{i}
$$

This equation just states that any small perturbation $\mathcal{O}$ can be expanded in the generators of translations along the local reference frame defined by $q_{i}, p_{i}$. Evolving in time, one observes

$$
\mathcal{O}(-t)=\sum_{i} \hat{p}_{i}(-t) \delta q_{i}-\hat{q}_{i}(-t) \delta p_{i}=\sum_{i} \hat{p}_{i} \delta q_{i}(t)-\hat{q}_{i} \delta p_{i}(t)
$$

where $\delta q_{i}(t)$ and $\delta p_{i}(t)$ are determined by a linearized equation of the type (4.55) associated to the quantum phase space. This is analyzed in a specific generic example in [15]. It follows that the growth properties of the perturbation $\mathcal{O}$ are controlled by the Jacobian matrix defining the chaotic process in the quantum phase space, and vice versa.

Classical chaos in AdS/CFT. In the context of the AdS/CFT correspondence, CFT perturbations $e^{i \mathcal{O}}|\psi\rangle$ generated by operators $\mathcal{O}$ with large conformal dimension can be described by freely falling particles in the bulk geometry dual to $|\psi\rangle$, in the semiclassical approximation [51-56]. At high energies, the bulk geometry involves a black hole with the temperature related to the energy of the state by the usual thermodynamic relation.

It was realized in [33-35] that the optical metric defined by

$$
d s_{\text {optical }}^{2} \equiv \frac{d s^{2}}{\left|g_{00}\right|},
$$

\footnotetext{
${ }^{21}$ There is a missing phase in the equation, due to the non-commutativity between $q$ and $p$. It is not included here because it is second order in the infinitesimal perturbations $\delta q, \delta p$.
} 
has an insightful structure in the near horizon region of a black hole. Concretely, for a black hole in $d+1$ dimensions we have that near the horizon

$$
d s_{\text {optical }}^{2} \approx-d t^{2}+\left(\frac{\beta}{2 \pi}\right)^{2} d s_{\mathbf{H}^{d}}^{2},
$$

where

$$
d s_{\mathbf{H}^{d}}^{2}=\frac{d y^{2}+d x_{\perp}^{2}}{y^{2}},
$$

is the metric of the euclidean hyperboloid with unit radius. Therefore, near the horizon, where particles become effectively massless [35], particle trayectories are controlled by geodesics on a hyperbolic space with radius of curvature given by $R=\beta / 2 \pi$. These geodesics are known to display chaotic properties. In fact, compact hyperbolic spaces are examples of hard chaos, and the geodesic deviation growth is characterized by a Lyapunov exponent given by $\lambda=1 / R=2 \pi / \beta$.

With the construction developed in previous sections, particularly in section 3 , we can now understand the emergence of this classical chaos. The hyperbolic geodesic deviation rests on the conformal transformation from the near horizon Rindler geometry to the hyperbolic one. It therefore secretly rests on the emergent Poincaré symmetries described above. The fact that Minkowski energies and radial momenta grow exponentially in the Rindler frame with Lyapunov exponent $\lambda=2 \pi / \beta$ is mapped, in the optical frame, to the fact that perturbations in the transverse direction grow exponentially fast with the same Lyapunov exponent. This is because it is the inverse of $\left|g_{00}\right|$ which grows exponentially fast as we approach the horizon. Such conformal transformation from the Rindler frame to the optical one was studied at the classical level and also in the QFT setup in [35, 57]. Having constructed the Poincaré symmetries above from the structure of the Heisenberg time evolution, such conformal transformation to the optical frame can be constructed as well, exactly as if we were studying free QFT in the Minkowski/Rindler scenario. In this way, the chaotic properties of the optical metric are thus recovered, and are transparently seen to be fundamentally attached to the validity of the equivalence principle near the horizon.

\section{Discussion}

We have considered the problem of operator growth in large- $\mathrm{N}$ gauge theories at finite temperature. We framed the problem as that of understanding the operators

$$
\mathcal{O}_{n} \equiv[H, \cdots,[H, \mathcal{O}] \cdots]
$$

where $H$ is the Hamiltonian. This is most natural since the expansion of Heisenberg time evolution in powers of time (1.2) teaches us these are the only operators with whom the initial operator mixes over time.

In section 2, we argued that the expression

$$
\mathcal{O}_{n}(t)=\int_{\omega>0} \frac{d \omega d^{d-1} \vec{k}}{(2 \pi)^{d}}\left((-\omega)^{n} \mathcal{O}_{\omega, \vec{k}} e^{-i \omega t+i \vec{k} \vec{x}}+\omega^{n} \mathcal{O}_{\omega, \vec{k}}^{\dagger} e^{i \omega t-i \vec{k} \vec{x}}\right)
$$


together with linearity, large- $\mathrm{N}$ factorization, the fact that most eigenstates at a given temperature behave as thermal states (ETH) and the correlation functions of the field modes $\mathcal{O}_{\omega, \vec{k}}$ and $\mathcal{O}_{\omega, \vec{k}}^{\dagger}$, given by (2.6), completely specify the action of these operators in most interesting states, up to $1 / N$ corrections. Hence, (5.2) determines the time evolution of the operator $\mathcal{O}(t, \vec{x})$. A similar statement holds for modular time evolution as well.

A first interesting insight is that any notion of operator growth should be determined by the 2-pt function at this order. Given the relation found between operator growth and four-point functions at an infinite temperature in SYK [1], one might hope for a non-trivial relation between the two-point function and the connected four-point function in generic large- $\mathrm{N}$ theories. We leave this interesting observation for future work.

In section 3, we constructed an emergent bulk Poincaré algebra (3.28) as the first application of our proposed solution. This was achieved by the known doubling of the modes appearing in large- $\mathrm{N}$ theories [25]. This algebra is related to the near horizon Rindler behaviour of thermal horizons. Albeit we have focused on the conventional modes, defined by means of the Hamiltonian of the large-N theory, the construction can be easily extended to modular time evolution, by using the modular modes (2.17) instead of the conventional ones. If large- $\mathrm{N}$ factorization holds, we can again find renormalized modes satisfying the algebra of free creation and annihilation operators, and proceed with the construction of an emergent Poincaré algebra. It would be interesting to develop the arguments given here in relation to the recent results in [39], based on prior work [58], where such Poincaré algebra emerges from a local bulk perspective due to the limiting modular evolution behaviour, making the latter much closer to Equivalence Principle considerations.

Albeit the simplicity of the previous solution contrasts with the expected complexity of the problem, in section 4, we analyzed several existent notions of operator growth and size existent in the literature from the perspective presented here. These include number operators, energy measures, the recursion method in condensed matter physics, and the approach to quantum chaos based on quantum circuit complexity. All of them can be considered in detail, and analytically, in the basis of field modes. We have seen that the different approaches are just variations over a common theme: the evolution in time of the initial operator $\mathcal{O}$, which is fully characterized by expression (2.2), at leading order. We made proposals for operator size in large-N QFTs by noticing that the GNS construction maps operator evolution to conventional state evolution in the GNS Hilbert space, and also derived an explicit relation between operator complexity and circuit complexity in eq. (4.54). In the large-N limit, all such notions are functionals of the two-point function alone. ${ }^{22}$

It is an important open problem to understand how to systematically incorporate $1 / N$ corrections to our discussion. Given our approach, this is not an intrinsic problem attached to operator growth, but it is generic to large- $\mathrm{N}$ gauge theories including holographic ones if one is interested in a bulk interpretation of these statements. See [22] for a recent discussion on how to incorporate these corrections in the bulk for some choices of operator size.

\footnotetext{
${ }^{22}$ The dependence of operator growth on the two-point function was noticed in ref. [46], for the case of the operator growth of a "complex" operator in the vacuum.
} 
We finish by stressing a point made in the introduction: our work neatly shows that any notion of operator growth has an equivalent formulation both in the bulk and the boundary theories, in the context of large-N holographic theories. This is just a consequence of the equality between bulk and boundary Hilbert space and Hamiltonians. In our setup, on a technical level, this is transparent due to the work in bulk reconstruction relating bulk field modes with boundary modes $\mathcal{O}_{\omega, \vec{k}}, \mathcal{O}_{\omega, \vec{k}}^{\dagger}$, and their mirror partners, mainly following [25-27].

\section{Acknowledgments}

We thank José Barbón, Pablo Bueno, Horacio Casini and Simon Ross for useful discussions. We also want to thank the Kavli Institute for Theoretical Physics and the Higgs Centre for Theoretical Physics for hospitality and financial support during the workshops "Chaos and Order" and "Recent development in holography", respectively. The work of JMM was supported by the Simons foundation through the It From Qubit Simons collaboration.

\section{A The Gelfand-Naimark-Segal (GNS) construction}

The GNS construction $[59,60]$ generates a Hilbert space $\mathcal{H}_{\omega}$ from an abstract $C^{\star}$-algebra $\mathcal{A}$ and a linear functional (state) $\omega$ from $\mathcal{A}$ to $\mathbb{C}$, together with a representation of the algebra $\pi(\mathcal{A})$ acting on it. In this appendix, we review its main ideas following closely [37].

A $C^{\star}$-algebra $\mathcal{A}$ is a set of objects such that if $A, B \in \mathcal{A}$ and $a, b \in \mathbb{C}$, then the linear combination $a A+b B \in \mathcal{A}$. Furthermore, there exists a map $A \mapsto A^{\dagger}, \forall A \in \mathcal{A}$ being an involution and satisfying

$$
(A B)^{\dagger}=B^{\dagger} A^{\dagger}, \quad(a A)^{\dagger}=a^{\star} A^{\dagger} \quad \forall A, B \in \mathcal{A}, \quad \forall a \in \mathbb{C}
$$

Up to topological requirements, see [37] for a more detailed account, $\mathcal{A}$ is called a von Neumann algebra if it further contains the identity. From now on we consider von Neumann algebras only.

States $\omega$ are positive and normalized linear functionals from $\mathcal{A}$ to $\mathbb{C}$ satisfying

$$
\begin{aligned}
\omega(a A+b B) & =a \omega(A)+b \omega(B), \\
\omega\left(A^{\star} A\right) & \geq 0 \\
\omega(\mathbb{1}) & =1 .
\end{aligned}
$$

Hilbert spaces $\mathcal{H}$ are vector spaces with an inner product mapping any pair of elements $|w\rangle,|v\rangle \in \mathcal{H}$ to a complex number $\langle v \mid w\rangle \in \mathbb{C}$ satisfying

$$
\begin{aligned}
\langle v \mid w\rangle & =\langle w \mid v\rangle^{\star}, \\
\left\langle a v_{1}+b v_{2} \mid w\right\rangle & =a^{\star}\left\langle v_{1} \mid w\right\rangle+b^{\star}\left\langle v_{2} \mid w\right\rangle, \\
|\langle v \mid v\rangle|^{2} & \geq 0
\end{aligned}
$$

where the last line is only saturated for $|v\rangle=0$. 
Let the algebra $\mathcal{A}$ be an algebra of operators. Since the algebra $\mathcal{A}$ is already a vector space over $\mathbb{C}$, to become a Hilbert space it requires an inner product. This can be defined using the state $\omega$ as

$$
\langle A \mid B\rangle=\omega\left(A^{\dagger} B\right),
$$

where we used the standard notation in quantum mechanics $|A\rangle$ to refer to the vector in $\mathcal{H}$ associated with the operator $A \in \mathcal{A}$.

This inner product satisfies all the requirements (A.3) except for the existence of nonzero operators $W$ satisfying $\omega\left(W^{\dagger} W\right)=0$. The set of such operators $\mathcal{I}$ is a left ideal in $\mathcal{A}$, the so called Gelfand ideal of the state $\omega$, i.e a linear subspace of $\mathcal{A}$ that is stable under multiplication by any element $A \in \mathcal{A}$ from the left

$$
W \in \mathcal{I}, \quad A \in \mathcal{A} \Rightarrow A W \in \mathcal{I} .
$$

The GNS construction defines the Hilbert space $\mathcal{H}_{\omega}$ as the quotient of $\mathcal{H}$ by the ideal $\mathcal{I}$, i.e. $\mathcal{H}_{\omega} \equiv \mathcal{A} / \mathcal{I}{ }^{23}$ Vectors $|[A]\rangle \in \mathcal{H}_{\omega}$ correspond to equivalence classes of operators in the algebra of the form $A+\mathcal{I}$ and such classes do not depend on the representative.

GNS induces a representation $\pi_{\omega}$ of $\mathcal{A}$ acting on $\mathcal{H}_{\omega}$ by the product in the algebra $\mathcal{A}$

$$
\pi_{\omega}(A)|[B]\rangle=|[A B]\rangle .
$$

A consequence of this construction is that the identity class $|\Omega\rangle \equiv|[\mathbb{1}]\rangle$ vector can be associated to the starting state $\omega$ since

$$
\omega(A)=\langle\Omega|A| \Omega\rangle
$$

\section{A.1 GNS of the thermal state}

When the GNS construction is considered for finite-dimensional algebras $\mathcal{A}$ containing bounded operators, the identity operator $\mathbb{1}$ can be understood as the maximally entangled density matrix (up to normalization) and the inner product (A.4) can be taken as

$$
\langle A \mid B\rangle=\frac{1}{Z} \operatorname{Tr}\left(A^{\dagger} B\right)
$$

where $Z \equiv \operatorname{Tr}(\mathbb{1})$. There is no Gelfand ideal $\mathcal{I}$ in this case. Hence, there exists an isomorphism between $\mathcal{A}$ and $\mathcal{H}_{\omega}$. The same conclusion holds when one replaces $\mathbb{1}$ with $\kappa=\rho_{\beta}^{1 / 2}$, where $\rho_{\beta}$ is the Boltzmann finite temperature density matrix (or any density matrix of full rank).

Besides the GNS representation $\pi(\mathcal{A})$

$$
\pi(A)|\kappa\rangle \equiv|A \kappa\rangle
$$

there exists the conjugate representation $\bar{\pi}(\mathcal{A})$, defined by

$$
\bar{\pi}(A)|\kappa\rangle \equiv\left|\kappa A^{\dagger}\right\rangle .
$$

\footnotetext{
${ }^{23}$ More precisely $\mathcal{H}_{\omega}$ is defined as the completion of $\mathcal{A} / \mathcal{I}$ with respect to the norm topology.
} 
These are equivalent because there exists an anti-unitary operator $J$ acting on $\mathcal{H}_{\omega}$ satisfying

$$
J|A \kappa\rangle=\left|\kappa A^{\dagger}\right\rangle \quad \text { with } \quad J^{2}=1
$$

implying

$$
J \pi(A) J=\bar{\pi}(A) .
$$

It also follows from these expressions that

$$
\omega(A)=\langle\kappa|\pi(A)| \kappa\rangle=\langle\kappa|\bar{\pi}(A)| \kappa\rangle^{*} .
$$

Since $|\kappa\rangle$ is invariant under time evolution, ${ }^{24}$ we can now easily define unitary evolution $U_{t}$ in all states of the representation by

$$
U_{t} \pi(A)|\kappa\rangle=\pi\left(A_{t}\right)|\kappa\rangle, \quad U_{t} \bar{\pi}(A)|\kappa\rangle=\bar{\pi}\left(A_{t}\right)|\kappa\rangle .
$$

It helps to disentangle the meaning of these definitions to explicitly write the unitary evolution as

$$
U_{t}=\pi\left(e^{i H t}\right) \bar{\pi}\left(e^{i H t}\right) .
$$

We can then check the definition (A.14)

$$
U_{t} \pi(A)|\kappa\rangle=U_{t}|A \kappa\rangle=\left|e^{i H t} A \kappa e^{-i H t}\right\rangle=\left|e^{i H t} A e^{-i H t} \kappa\right\rangle=\left|A_{t} \kappa\right\rangle=\pi\left(A_{t}\right)|\kappa\rangle
$$

is satisfied. Given this representation, it is natural to introduce the full hamiltonian as $H_{\mathrm{F}}=\pi(H)-\bar{\pi}(H)$. Using $\kappa=\rho_{\beta}^{1 / 2}=Z^{-1 / 2} e^{-\beta H / 2}$, it follows

$$
J e^{-\beta H_{\mathrm{F}} / 2} \pi(A)|\kappa\rangle=J e^{-\beta H_{\mathrm{F}} / 2}|A \kappa\rangle=J|\kappa A\rangle=J \bar{\pi}\left(A^{\dagger}\right)|\kappa\rangle=\pi\left(A^{\dagger}\right)|\kappa\rangle .
$$

It is interesting to single out the equality from the first term to the fourth term. Multiplying from the left both side by $J$ and moving the right hand side to the left we obtain

$$
\left(e^{-\beta H_{\mathrm{F}} / 2} \pi(A)-\bar{\pi}\left(A^{\dagger}\right)\right)|\kappa\rangle=0,
$$

which is the (generalized) origin of the known relations (3.6) and (3.22), associated to free QFT and large-N theories.

As stressed through the article, one general lesson is that operator evolution can be seen as a conventional state evolution through the GNS construction. The generator of the GNS unitary evolution, the GNS Hamiltonian, is what in the condensed matter community is called the Liouvillian [18], see [13, 14] for recent applications of such approach. Furthermore, the GNS construction points out the subtlety of the Gelfand ideal, stressing why states with full rank are convenient, and allows a direct application into QFT since it is valid for all types of algebras.

\footnotetext{
${ }^{24}$ For a general full rank state $\rho$, the evolution which is naturally defined by this construction is the so-called modular evolution. If $\rho=e^{-H}$, with $H$ the modular Hamiltonian, then $H$ is the generator of modular time evolution.
} 


\section{A.2 Inner products in the space of operators}

In the previous GNS construction, one starts with a natural inner product on the space of operators, such as (A.8) or (A.13). This choice of inner product is not unique. Denoting a general inner product by $(A, B)$, in [18] the following family is considered

$$
(A, B)=\frac{1}{\beta} \int_{0}^{\beta} d \lambda g(\lambda)\left\langle e^{\lambda H} A^{\dagger} e^{-\lambda H} B\right\rangle_{\beta}-\left\langle A^{\dagger}\right\rangle_{\beta}\langle B\rangle_{\beta},
$$

where $\langle A\rangle_{\beta} \equiv \operatorname{Tr}\left(\rho_{\beta} A\right)$ and $g(\lambda)$ is any function satisfying:

$$
g(\lambda) \geq 0 \quad g(\beta-\lambda)=g(\lambda) \quad \frac{1}{\beta} \int_{0}^{\beta} d \lambda g(\lambda)=1 .
$$

Examples of such functions are

$$
g(\lambda)=\frac{1}{2} \beta[\delta(\lambda)+\delta(\beta-\lambda)], \quad g(\lambda)=\delta(\beta / 2-\lambda),
$$

for which the inner product reduces to

$$
(A, B)=\frac{1}{2}\left\langle A^{\dagger} B+B A^{\dagger}\right\rangle-\left\langle A^{\dagger}\right\rangle\langle B\rangle, \quad(A, B)=\left\langle e^{\beta H / 2} A^{\dagger} e^{-\beta H / 2} B\right\rangle_{\beta}-\left\langle A^{\dagger}\right\rangle\langle B\rangle,
$$

respectively. In this section we want to describe the status of this big family (A.19) of inner products. In particular, we show below how they change the specific functional describing the chaotic growth. Indeed faster growths than the chaos bound can be obtained, albeit there is no surprise here, since one can actually relate all the inner products (A.19) to the one defined by $g(\lambda)=\beta \delta(\beta / 2-\lambda)$, as we show below.

To test the dependence on the inner product we can analyze the basic quantity controlling the growth, which is the return probability

$$
p(t) \equiv|(\mathcal{O}(t) \mid \mathcal{O}(0))|^{2} .
$$

Using the previous inner products we thus need to analyze ${ }^{25}$

$$
(\mathcal{O}(t), \mathcal{O}(0))=\frac{1}{\beta} \int_{0}^{\beta} d \lambda g(\lambda)\left\langle e^{\lambda H} \mathcal{O}(t) e^{-\lambda H} \mathcal{O}\right\rangle_{\beta}=\frac{1}{\beta} \int_{0}^{\beta} d \lambda g(\lambda)\langle\mathcal{O}(t-i \lambda) \mathcal{O}\rangle_{\beta},
$$

and such function is completely determined equivalently by its Fourier transform

$$
R(\omega)=\int e^{i \omega t}(\mathcal{O}(t), \mathcal{O}(0)) .
$$

To find such Fourier transform first consider:

$$
G_{\lambda}(\omega) \equiv \int e^{i \omega t}\langle\mathcal{O}(t-i \lambda) \mathcal{O}\rangle_{\beta}
$$

\footnotetext{
${ }^{25}$ We assume the operator $\mathcal{O}$ to have vanishing one point function for visual clarity. Including in the discussion one-point functions is trivial since they do not depend on time.
} 
The expectation value $\langle\mathcal{O}(t) \mathcal{O}\rangle_{\beta}$ can be analytically continued to imaginary times for $0>\operatorname{Im}(t)>-\beta$. Call $F(z)$ such a unique function in the strip. We can consider the following integrals of such function:

$$
\int_{\mathcal{C}_{\lambda}} F(z) e^{i z \omega} d z
$$

where $\mathcal{C}_{\lambda}$ is a path parallel to the real time axis, shifted by $-i \lambda$. Due to the absence of poles or singularities in such a region, Cauchy's theorem implies:

$$
\int_{\mathcal{C}_{\lambda}} F(z) e^{i z \omega} d z=\int_{\mathcal{C}_{\lambda^{\prime}}} F(z) e^{i z \omega} d z
$$

This implies that:

$$
\int_{-\infty}^{\infty} F(t-i \lambda) e^{i(t-i \lambda) \omega} d t=\int_{-\infty}^{\infty} F\left(t-i \lambda^{\prime}\right) e^{i\left(t-i \lambda^{\prime}\right) \omega}
$$

Looking at (A.26), we conlude that

$$
e^{\lambda \omega} G_{\lambda}(\omega)=e^{\lambda^{\prime} \omega} G_{\lambda^{\prime}}(\omega)
$$

This relation is important, since it says that knowing the exact Fourier transform at some imaginary time $\lambda$ is equivalent to knowing it at all complexified times in the holomorphic strip. In particular, for 2d CFT's, we have the exact results for $\lambda=\beta / 2$, so that for general $0<\lambda<\beta$ :

$$
G_{\lambda}(\omega)=e^{-\lambda \omega} e^{\beta \omega / 2} G_{\beta / 2}(\omega)
$$

The Fourier transform of the autocorrelation function ends up being:

$$
R(\omega)=e^{\beta \omega / 2} G_{\beta / 2}(\omega) \frac{1}{\beta} \int_{0}^{\beta} d \lambda g(\lambda) e^{-\lambda \omega},
$$

which is a simple functional of the information encoded in the inner product defined by $g(\lambda)=\beta \delta(\beta / 2-\lambda)$.

The previous relation implies that all inner products have slower decay tails of $R(\omega)$ at large $\omega$ than the choice $g(\lambda)=\beta \delta(\beta / 2-\lambda)$. The choice $g(\lambda)=\beta \delta(\beta / 2-\lambda)$ is indeed the one considered in [13], which in chaotic QFT leads to a exponential growth with Lyapunov exponent $\lambda=2 \pi / \beta$. Therefore, we conclude that all other choices display stronger Lanczos growths than the one expected by the chaos bound. We point out the existence of the results found in [47], in the context of OTOCs, which show that different choices of euclidean separations in the 4-pt functions lead to faster growths than the chaos bound [8]. 


\section{B Solving the recurrence relation}

In this appendix we complete the proof of the solution of the Lanczos recursion method when applied to large-N theories. Given $P_{0, j}$ and $Q_{0, j}$ in (4.38), the ansatz (4.42) reproduces correctly $P_{1, j}$ and $Q_{1, j}$. Assuming (4.42) holds for $s$, we want to show $P_{s+1, j}$ and $Q_{s+1, j}$ satisfy (4.42). Let us use the first equation in (4.41) to compute $P_{s+1, j}$

$$
\begin{aligned}
P_{s+1, j}= & -\omega_{j} Q_{s, j}+\Delta_{2 s+1} P_{s, j} \\
= & \sum_{m=0}^{s}(-1)^{s+1} \omega_{j}^{2(m+1)} \sum_{i_{1}=1}^{2(m+1)} \Delta_{i_{1}} \sum_{i_{2}=i_{1}+2}^{2(m+2)} \Delta_{i_{2}} \ldots \sum_{i_{s-m}=i_{s-m-1}+2}^{2 s} \Delta_{i_{s-m}} \\
& +\Delta_{2 s+1} \sum_{r=0}^{s}(-1)^{r} \omega_{j}^{2 r} \sum_{i_{1}=1}^{2 r+1} \Delta_{i_{1}} \sum_{i_{2}=i_{1}+2}^{2 r+3} \Delta_{i_{2}} \cdots \sum_{i_{s-r}=i_{s-r-1}+2}^{2 s-1} \Delta_{i_{s-r}} \\
= & (-1)^{s+1} \omega_{j}^{s+1}+\sum_{r=1}^{s}(-1)^{r} \omega_{j}^{2 r} \sum_{i_{1}=1}^{2 r} \Delta_{i_{1}} \sum_{i_{2}=i_{1}+2}^{2(r+1)} \Delta_{i_{2}} \ldots \sum_{i_{s-m}=i_{s+1-r}+2}^{2 s} \Delta_{i_{s-m}} \\
& +\Delta_{2 s+1} \sum_{r=0}^{s}(-1)^{r} \omega_{j}^{2 r} \sum_{i_{1}=1}^{2 r+1} \Delta_{i_{1}} \sum_{i_{2}=i_{1}+2}^{2 r+3} \Delta_{i_{2}} \ldots \sum_{i_{s-r}=i_{s-r-1}+2}^{2 s-1} \Delta_{i_{s-r}} .
\end{aligned}
$$

To derive the third equality, we relabelled $m+1=r$ and wrote the $r=s+1$ term separately, i.e. the only one involving no $\Delta$ s. Notice that the only $\omega_{j}^{0}$ term comes from $r=0$ in the last line and reproduces the right answer $\Delta_{2 s+1} \Delta_{1} \Delta_{3} \ldots \Delta_{2 s-1}$. Hence, given an index $r=\{1,2, \ldots s\}$, the task is to show

$$
\sum_{i_{1}=1}^{2 r+1} \Delta_{i_{1}} \sum_{i_{2}=i_{1}+2}^{2 r+3} \Delta_{i_{2}} \cdots \sum_{i_{s+1-r}=i_{s-r-1}+2}^{2 s+1} \Delta_{i_{s+1-r}}
$$

equals the sum of the coefficients in the last two lines of (B.1) for a given $r$. The key point is the different upper limit in the last sum in (B.2) being $2 s+1$. Indeed, when $i_{s+1-r} \neq 2 s+1$, the contribution from (B.2) equals the first line in (B.1). Hence, we are left to show the contribution from $i_{s+1-r}=2 s+1$ in (B.2)

$$
\Delta_{2 s+1} \sum_{i_{1}=1}^{2 r+1} \Delta_{i_{1}} \sum_{i_{2}=i_{1}+2}^{2 r+3} \Delta_{i_{2}} \cdots \sum_{i_{s-r}=i_{s-r-1}+2}^{2 s-1} \Delta_{i_{s-r}}
$$

equals the last line in (B.1), which it does.

We are left to check $Q_{s+1, j}$ satisfies

$$
Q_{s+1, j}=\omega_{j} \sum_{m=0}^{s+1}(-1)^{m} \omega_{j}^{2 m} \sum_{i_{1}=1}^{2(m+1)} \Delta_{i_{1}} \sum_{i_{2}=i_{1}+2}^{2(m+2)} \Delta_{i_{2}} \cdots \sum_{i_{s+1-m}=i_{s-m}+2}^{2(s+1)} \Delta_{i_{s+1-m}}
$$


having assumed $P_{s+1, j}$ and $Q_{s, j}$ do satisfy (4.42). Using the second recursion relation (4.42), we can write $Q_{s+1, j}$ as

$$
\begin{aligned}
& \omega_{j} P_{s+1, j}+\Delta_{2(s+1)} Q_{s, j}= \\
& \omega_{j} \sum_{m=0}^{s+1}(-1)^{m} \omega_{j}^{2 m} \sum_{i_{1}=1}^{2 m+1} \Delta_{i_{1}} \sum_{i_{2}=i_{1}+2}^{2 m+3} \Delta_{i_{2}} \ldots \sum_{i_{s+1-m}=i_{s-m}+2}^{2 s+1} \Delta_{i_{s+1-m}} \\
& +\Delta_{2(s+1)} \omega_{j} \sum_{m=0}^{s}(-1)^{m} \omega_{j}^{2 m} \sum_{i_{1}=1}^{2(m+1)} \Delta_{i_{1}} \sum_{i_{2}=i_{1}+2}^{2(m+2)} \Delta_{i_{2}} \ldots \sum_{i_{s-m}=i_{s-m-1}+2}^{2 s} \Delta_{i_{s-m}}
\end{aligned}
$$

Notice the second line already has the right functional dependence and the right number of sum terms, except for the fact that upper limits in the sums are off. In particular, the last index is not allowed to reach the maximal value $i_{s+1-m}=2(s+1)$. Consider the contribution from $\Delta_{2(s+1)}$ in (B.4)

$$
\Delta_{2(s+1)} \omega_{j} \sum_{m=0}^{s+1}(-1)^{m} \omega_{j}^{2 m} \sum_{i_{1}=1}^{2(m+1)} \Delta_{i_{1}} \ldots \sum_{i_{s-m}=i_{s-m-1}+2}^{2 s} \Delta_{i_{s-m}} .
$$

In our conventions, the term $m=s+1$ vanishes, be definition. The remaining terms match $\Delta_{2(s+1)} Q_{s, j}$ above. The only remaining question is whether the terms in $Q_{s+1, j}$ not including $\Delta_{2(s+1)}$

$$
\omega_{j} \sum_{m=0}^{s+1}(-1)^{m} \omega_{j}^{2 m} \sum_{i_{1}=1}^{2(m+1)} \Delta_{i_{1}} \sum_{i_{2}=i_{1}+2}^{2(m+2)} \Delta_{i_{2}} \cdots \sum_{i_{s+1}}^{2 s+1} \Delta_{i_{s+1-m}}
$$

equal $\omega_{j} P_{s+1, j}$. Since the upper limit in $i_{s+1-m}$ was reduced to $2 s+1$, the remaining upper limits should also be decreased by one, finishing the proof.

Open Access. This article is distributed under the terms of the Creative Commons Attribution License (CC-BY 4.0), which permits any use, distribution and reproduction in any medium, provided the original author(s) and source are credited.

\section{References}

[1] D.A. Roberts, D. Stanford and A. Streicher, Operator growth in the SYK model, JHEP 06 (2018) 122 [arXiv: 1802.02633] [INSPIRE].

[2] X.-L. Qi and A. Streicher, Quantum Epidemiology: Operator Growth, Thermal Effects and SYK, JHEP 08 (2019) 012 [arXiv:1810.11958] [INSPIRE].

[3] S. Sachdev and J. Ye, Gapless spin fluid ground state in a random, quantum Heisenberg magnet, Phys. Rev. Lett. 70 (1993) 3339 [cond-mat/9212030] [INSPIRE].

[4] A. Kitaev, A simple model of quantum holography (part 1), talk at KITP, April 7, 2015, http://online.kitp.ucsb.edu/online/entangled15/kitaev/.

[5] A. Kitaev, A simple model of quantum holography (part 2), talk at KITP, May 27, 2015, http://online.kitp.ucsb.edu/online/entangled15/kitaev2/. 
[6] A.I. Larkin and Y.N. Ovchinnikov, Quasiclassical Method in the Theory of Superconductivity, JETP 28 (1969) 1200.

[7] S.H. Shenker and D. Stanford, Stringy effects in scrambling, JHEP 05 (2015) 132 [arXiv: 1412.6087] [INSPIRE].

[8] J. Maldacena, S.H. Shenker and D. Stanford, A bound on chaos, JHEP 08 (2016) 106 [arXiv: 1503.01409] [INSPIRE].

[9] L. Susskind, Why do Things Fall?, arXiv:1802.01198 [INSPIRE].

[10] J.M. Magán, Black holes, complexity and quantum chaos, JHEP 09 (2018) 043 [arXiv: 1805.05839] [INSPIRE].

[11] H.W. Lin, J. Maldacena and Y. Zhao, Symmetries Near the Horizon, JHEP 08 (2019) 049 [arXiv:1904.12820] [INSPIRE].

[12] J.L.F. Barbón, J. Martín-García and M. Sasieta, Momentum/Complexity Duality and the Black Hole Interior, arXiv:1912.05996 [INSPIRE].

[13] D.E. Parker, X. Cao, A. Avdoshkin, T. Scaffidi and E. Altman, A Universal Operator Growth Hypothesis, Phys. Rev. X 9 (2019) 041017 [arXiv: 1812.08657] [InSPIRE].

[14] J.L.F. Barbón, E. Rabinovici, R. Shir and R. Sinha, On The Evolution Of Operator Complexity Beyond Scrambling, JHEP 10 (2019) 264 [arXiv:1907.05393] [INSPIRE].

[15] P. Bueno, J.M. Magán and C.S. Shahbazi, Complexity measures in QFT and constrained geometric actions, arXiv:1908.03577 [INSPIRE].

[16] M.A. Nielsen, A geometric approach to quantum circuit lower bounds, quant-ph/0502070.

[17] M.R. Dowling and M.A. Nielsen, The geometry of quantum computation, quant-ph/0701004.

[18] V. Viswanath and G. Müller, The Recursion Method: Application to Many-Body Dynamics, vol. 23, Springer Science \& Business Media, (2008).

[19] A. Streicher, SYK Correlators for All Energies, JHEP 02 (2020) 048 [arXiv:1911.10171] [INSPIRE].

[20] A. Lucas, Non-perturbative dynamics of the operator size distribution in the Sachdev-Ye-Kitaev model, arXiv: 1910.09539 [INSPIRE].

[21] Y.D. Lensky, X.-L. Qi and P. Zhang, Size of bulk fermions in the SYK model, arXiv:2002.01961 [INSPIRE].

[22] A. Mousatov, Operator Size for Holographic Field Theories, arXiv:1911.05089 [InSPIRE].

[23] G. 't Hooft, A Planar Diagram Theory for Strong Interactions, Nucl. Phys. B 72 (1974) 461 [inSPIRE].

[24] S. El-Showk and K. Papadodimas, Emergent Spacetime and Holographic CFTs, JHEP 10 (2012) 106 [arXiv:1101.4163] [InSPIRE].

[25] K. Papadodimas and S. Raju, An Infalling Observer in AdS/CFT, JHEP 10 (2013) 212 [arXiv: 1211.6767] [INSPIRE].

[26] K. Papadodimas and S. Raju, Black Hole Interior in the Holographic Correspondence and the Information Paradox, Phys. Rev. Lett. 112 (2014) 051301 [arXiv:1310.6334] [INSPIRE].

[27] K. Papadodimas and S. Raju, State-Dependent Bulk-Boundary Maps and Black Hole Complementarity, Phys. Rev. D 89 (2014) 086010 [arXiv:1310.6335] [InSPIRE]. 
[28] J. De Boer, R. Van Breukelen, S.F. Lokhande, K. Papadodimas and E. Verlinde, Probing typical black hole microstates, JHEP 01 (2020) 062 [arXiv: 1901.08527] [INSPIRE].

[29] J.M. Maldacena, The large $N$ limit of superconformal field theories and supergravity, Int. J. Theor. Phys. 38 (1999) 1113 [hep-th/9711200] [INSPIRE].

[30] S.S. Gubser, I.R. Klebanov and A.M. Polyakov, Gauge theory correlators from noncritical string theory, Phys. Lett. B 428 (1998) 105 [hep-th/9802109] [INSPIRE].

[31] E. Witten, Anti-de Sitter space and holography, Adv. Theor. Math. Phys. 2 (1998) 253 [hep-th/9802150] [INSPIRE].

[32] M. Srednicki, Chaos and quantum thermalization, Phys. Rev. E 50 (1994) 888.

[33] J.L.F. Barbón and J.M. Magán, Chaotic Fast Scrambling At Black Holes, Phys. Rev. D 84 (2011) 106012 [arXiv: 1105.2581] [INSPIRE].

[34] J.L.F. Barbón and J.M. Magán, Fast Scramblers Of Small Size, JHEP 10 (2011) 035 [arXiv: 1106.4786] [INSPIRE].

[35] J.L.F. Barbón and J.M. Magán, Fast Scramblers, Horizons and Expander Graphs, JHEP 08 (2012) 016 [arXiv: 1204.6435] [INSPIRE].

[36] J. de Boer, R. Van Breukelen, S.F. Lokhande, K. Papadodimas and E. Verlinde, On the interior geometry of a typical black hole microstate, JHEP 05 (2019) 010 [arXiv: 1804.10580] [INSPIRE].

[37] R. Haag, Local quantum physics: Fields, particles, algebras, Springer, (1992).

[38] E. Witten, APS Medal for Exceptional Achievement in Research: Invited article on entanglement properties of quantum field theory, Rev. Mod. Phys. 90 (2018) 045003 [arXiv: 1803. 04993] [INSPIRE].

[39] J. De Boer and L. Lamprou, Holographic Order from Modular Chaos, arXiv:1912.02810 [INSPIRE].

[40] E. Witten, Anti-de Sitter space, thermal phase transition and confinement in gauge theories, Adv. Theor. Math. Phys. 2 (1998) 505 [hep-th/9803131] [INSPIRE].

[41] L.C.B. Crispino, A. Higuchi and G.E.A. Matsas, The Unruh effect and its applications, Rev. Mod. Phys. 80 (2008) 787 [arXiv:0710.5373] [INSPIRE].

[42] J.M. Maldacena, Eternal black holes in anti-de Sitter, JHEP 04 (2003) 021 [hep-th/0106112] [INSPIRE].

[43] W.G. Unruh, Notes on black hole evaporation, Phys. Rev. D 14 (1976) 870 [INSPIRE].

[44] A. Almheiri, X. Dong and D. Harlow, Bulk Locality and Quantum Error Correction in AdS/CFT, JHEP 04 (2015) 163 [arXiv:1411.7041] [INSPIRE].

[45] D. Harlow, TASI Lectures on the Emergence of Bulk Physics in AdS/CFT, PoS(TASI2017) 002 [arXiv: 1802.01040] [INSPIRE].

[46] J.M. Magán, Decoherence and microscopic diffusion at the Sachdev-Ye-Kitaev model, Phys. Rev. D 98 (2018) 026015 [arXiv:1612.06765] [InSPIRE].

[47] A. Romero-Bermúdez, K. Schalm and V. Scopelliti, Regularization dependence of the OTOC. Which Lyapunov spectrum is the physical one?, JHEP 07 (2019) 107 [arXiv:1903.09595] [INSPIRE]. 
[48] M.A. Nielsen, M.R. Dowling, M. Gu and A.C. Doherty, Quantum Computation as Geometry, Science 311 (2006) 1133 [quant-ph/0603161].

[49] A. Bernamonti, F. Galli, J. Hernandez, R.C. Myers, S.-M. Ruan and J. Simón, First Law of Holographic Complexity, Phys. Rev. Lett. 123 (2019) 081601 [arXiv: 1903.04511] [INSPIRE].

[50] A. Ashtekar and T.A. Schilling, Geometrical formulation of quantum mechanics, gr-qc/9706069 [INSPIRE].

[51] A.L. Fitzpatrick and J. Kaplan, Scattering States in AdS/CFT, arXiv:1104.2597 [INSPIRE].

[52] M. Nozaki, T. Numasawa and T. Takayanagi, Holographic Local Quenches and Entanglement Density, JHEP 05 (2013) 080 [arXiv: 1302.5703] [INSPIRE].

[53] K. Goto, M. Miyaji and T. Takayanagi, Causal Evolutions of Bulk Local Excitations from CFT, JHEP 09 (2016) 130 [arXiv: 1605.02835] [INSPIRE].

[54] K. Goto and T. Takayanagi, CFT descriptions of bulk local states in the AdS black holes, JHEP 10 (2017) 153 [arXiv:1704.00053] [INSPIRE].

[55] S. Terashima, AdS/CFT Correspondence in Operator Formalism, JHEP 02 (2018) 019 [arXiv:1710.07298] [INSPIRE].

[56] D. Berenstein and J. Simón, Localized states in global AdS space, Phys. Rev. D 101 (2020) 046026 [arXiv: 1910 . 10227] [INSPIRE].

[57] H. Casini, M. Huerta and R.C. Myers, Towards a derivation of holographic entanglement entropy, JHEP 05 (2011) 036 [arXiv:1102.0440] [INSPIRE].

[58] B. Czech, J. De Boer, D. Ge and L. Lamprou, A modular sewing kit for entanglement wedges, JHEP 11 (2019) 094 [arXiv: 1903. 04493] [INSPIRE].

[59] I. Gelfand and M. Neumark, On the imbedding of normed rings into the ring of operators in hilbert space, Rec. Math. [Mat. Sbornik] N.S. 12(54) (1943) 197.

[60] I.E. Segal, Irreducible representations of operator algebras, Bull. Am. Math. Soc. 53 (1947) 73. 